\title{
Hyperbolic metamaterials: fusing artificial structures to natural 2D materials
}

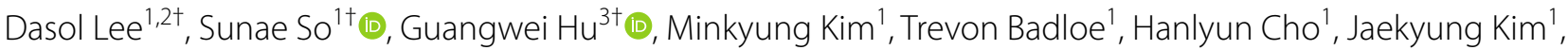 \\ Hongyoon Kim ${ }^{1}$, Cheng-Wei Qiü ${ }^{3 *}$ and Junsuk Rho ${ }^{1,4,5^{*}}$ (D)
}

\begin{abstract}
Optical metamaterials have presented an innovative method of manipulating light. Hyperbolic metamaterials have an extremely high anisotropy with a hyperbolic dispersion relation. They are able to support high-k modes and exhibit a high density of states which produce distinctive properties that have been exploited in various applications, such as super-resolution imaging, negative refraction, and enhanced emission control. Here, state-of-the-art hyperbolic metamaterials are reviewed, starting from the fundamental principles to applications of artificially structured hyperbolic media to suggest ways to fuse natural two-dimensional hyperbolic materials. The review concludes by indicating the current challenges and our vision for future applications of hyperbolic metamaterials.
\end{abstract}

Keywords: Hyperbolic metamaterials, High resolution optical imaging, Nanoscale lithography, Light propagation and manipulation, Spontaneous emission engineering, Natural 2D materials

\section{Introduction}

In the last decade, the ever increasing research and understanding of light-matter interactions has become a catalyst for the development of new technologies to control light. In particular, optical metamaterials can demonstrate unique optical properties that are not found in natural materials. The correct design and arrangement of sub-wavelength sized structures, known as meta-atoms, can be used to effectively control and manipulate lightmatter interactions. Innovations in nanofabrication and measurement methods have given rise to various applications such as negative refraction [1], metaholograms [2-13], and metalenses [14-20].

Among various metamaterials that have been realized to date, hyperbolic metamaterials (HMMs) have attracted great interest due to their highly anisotropic

\footnotetext{
*Correspondence: chengwei.qiu@nus.edu.sg; jsrho@postech.ac.kr

†Dasol Lee, Sunae So and Guangwei Hu contributed equally to this work 1 Department of Mechanical Engineering, Pohang University of Science and Technology (POSTECH), Pohang 37673, Republic of Korea

${ }^{3}$ Department of Electrical and Computer Engineering, National University of Singapore, Singapore 117583, Singapore

Full list of author information is available at the end of the article
}

characteristics [21-23]. The hyperbolic dispersion of HMMs is determined by the effective permittivity tensor where the principal components of the electric or magnetic fields have opposite signs. The relatively easy fabrication of multilayer or nanowire (NW) structures can yield HMMs that have a three-dimensional (3D) bulk response at optical frequencies. Besides, such materials can support the hyperbolic dispersion, where in principle the infinitely large unbounded momentum and thus the very high confinement of light can be allowed. Thus, such unique anisotropic properties have been applied to various distinguished applications including super-resolution imaging [24, 25], negative refraction [26, 27], and emission engineering $[28,29]$.

The propagation loss is one of the main limitations of bulk HMMs, due to the inevitable Ohmic loss of incorporated plasmonic materials, so methods to reduce it are being actively sought after. As a result, two-dimensional (2D) hyperbolic metasurfaces (HMSs) have been investigated, rendering the hyperbolic propagation and large confinement of light at the surface and not the bulk, and thus are less sensitive the loss and have shown great potentials as planar optical devices. Besides, some 
pristine 2D materials and bulk crystals can render the natural hyperbolic response, without the need of structuring, and have been explored as a powerful alternative candidate for the realization of HMMs and HMSs. Natural 2D hyperbolic materials have a homogeneous hyperbolic dispersion, so have been explored as a powerful alternative candidate for the realization of HMMs and HMSs. Numerous materials that exhibit hyperbolic dispersion at various wavelengths have been demonstrated, signalling the high potential of HMMs and HMSs for implementation in practical applications [30-33].

Herein, we first introduce the basic theory for the realization of HMMs. We will present details of hyperbolic dispersion, bulk HMMs, and planar 2D hyperbolic materials. Following the fundamental discussions, we will review the progress of applications of HMMs and HMSs, including the most recently reported papers and newly emerging fields, with emphasis on high-resolution optical imaging and nanoscale lithography, light propagation and manipulation, spontaneous emission engineering, sensors, and absorbers. Then we present the recent tremendous progress of natural hyperbolic materials that are key for further investigation to realize planar hyperbolic applications. Last, we conclude by highlighting the current challenges and suggesting an overview for future research on hyperbolic metamaterials. While following the existing framework, this review contains the latest publications that have not been reviewed elsewhere. We expect that this review will faithfully fill the gap with existing review articles and provide insight and potential of HMMs as a powerful tool for photonic devices.

\section{Theory: realization of hyperbolic metamaterials 2.1 Dispersion}

The concept of HMMs originates from optical crystals, where the effective permittivity $(\hat{\varepsilon})$ and permeability $(\hat{\mu})$ tensors of the material oriented along the principal axes are described as

$$
\begin{aligned}
& \hat{\varepsilon}=\varepsilon_{0}\left(\begin{array}{ccc}
\varepsilon_{x x} & 0 & 0 \\
0 & \varepsilon_{y y} & 0 \\
0 & 0 & \varepsilon_{z z}
\end{array}\right), \\
& \hat{\mu}=\mu_{0}\left(\begin{array}{ccc}
\mu_{x x} & 0 & 0 \\
0 & \mu_{y y} & 0 \\
0 & 0 & \mu_{z z}
\end{array}\right),
\end{aligned}
$$

where, $\varepsilon_{0}$ and $\mu_{0}$ are the permittivity and permeability of a vacuum, respectively. In general, materials are isotropic, which means that the permittivity and permeability components are equal in all directions $\left(\varepsilon_{x x}=\varepsilon_{y y}=\varepsilon_{z z}\right.$, $\mu_{x x}=\mu_{y y}=\mu_{z z}$ ). If one or more of these principal components is different, the material becomes either a uniaxial or biaxial anisotropic medium. Assuming that the optical axis (OA) is along the $z$ direction, an electric uniaxial medium has $\varepsilon_{x x}=\varepsilon_{y y} \neq \varepsilon_{z z}$, whereas a magnetic uniaxial medium has $\mu_{x x}=\mu_{y y} \neq \mu_{z z}$; and an electric biaxial medium has $\varepsilon_{x x} \neq \varepsilon_{y y} \neq \varepsilon_{z z}$, whereas a magnetic biaxial medium has $\mu_{x x} \neq \mu_{y y} \neq \mu_{z z}$. For simplicity, we now consider electric uniaxial material under transverse magnetic (TM) polarized light. The dispersion relation of the light in the medium with effective permittivity in Eq. (1) can be derived from Maxwell's equations as

$$
\frac{k_{x}^{2}+k_{y}^{2}}{\varepsilon_{z z}}+\frac{k_{z}^{2}}{\varepsilon_{x x}}=\frac{\omega^{2}}{c^{2}},
$$

where $k_{x}, k_{y}, k_{z}$ are the wave vector components along the $x, y$, and $z$ directions, respectively, $\omega$ is the angular frequency, and $c$ is the speed of light. In Eq. (2), the isofrequency contour (IFC) can be obtained at the intersection of the constant frequency plane and the dispersion surface. In general materials, the components of the effective permittivity all have the same sign, so the IFC is closed and forms a sphere or ellipsoid (Fig. 1a). Due to the bounded IFC, waves with large wave vectors become evanescent in those materials. In contrast, under the condition where the signs of two components of the permittivity are opposite $\left(\varepsilon_{z z} \cdot \varepsilon_{x x}<0\right)$, the IFC becomes an unbounded hyperboloid that can support high- $k$ waves $[21-23,34,35]$. These properties are not commonly obtained in nature $[33,36,37]$, but can be achieved in a fairly straightforward manner using metamaterials [1, 38-40]. HMMs are named after the hyperbolic topology of their IFC, from which their unique properties stem from. HMMs can be classified into two types according to the sign of their permittivity. Materials with $\varepsilon_{x x}=\varepsilon_{y y}>0, \varepsilon_{z z}<0$ have a predominantly dielectric nature, and are therefore called dielectric-type or TypeI HMMs (Fig. 1b); materials with $\varepsilon_{x x}=\varepsilon_{y y}<0, \varepsilon_{z z}>0$ have more metallic properties, and are subsequently known as metallic-type or Type-II HMMs (Fig. 1c). Because of their predominant metallic nature, Type-II HMMs show highly absorptive properties, and hence only support high- $k$ waves that have a cut-off spatial frequency. Magnetic HMMs [41-43] can also be achieved under transverse electric (TE) polarization by using a magnetic permeability tensor that has opposite signs in one or two directions.

\subsection{Bulk hyperbolic metamaterials}

A few naturally occurring hyperbolic materials have a strong anisotropy [33, 36, 37]. Examples include the crystal structures of graphite (C) [30], hexagonal boron nitride (hBN) [31], magnesium diboride $\left(\mathrm{MgB}_{2}\right)$ [32], calcite $\left(\mathrm{CaCO}_{3}\right)$ [44], and crystals with perovskite layers of cuprate and ruthenate [33]. In these structures, 


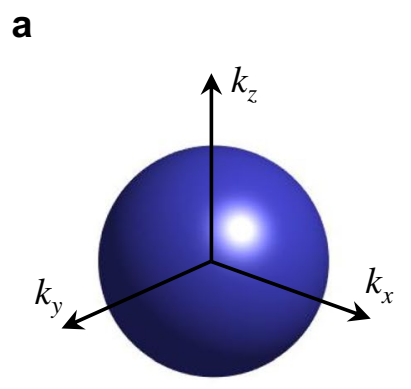

b

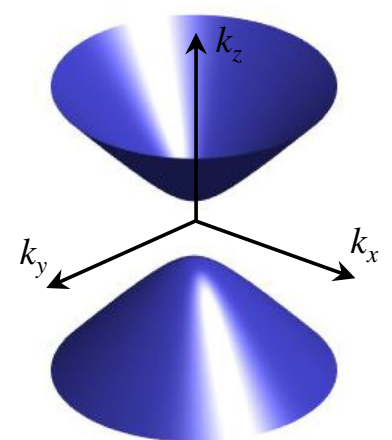

C

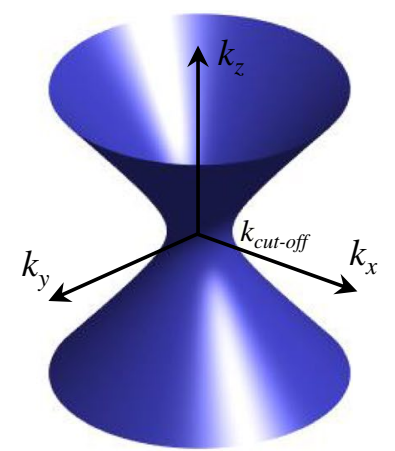

Fig. 1 Isofrequency contours. Isofrequency contours for a an isotropic medium, b a dielectric-type hyperbolic metamaterial (HMM), and c a metallic-type HMM

Table 1 Naturally occuring hypebolic materials and their operating wavelengths

\begin{tabular}{lll}
\hline \multicolumn{2}{c}{ Material } & Wavelength $[\boldsymbol{\mu m}]$ \\
\hline UV & Graphite $(\mathrm{C})[30]$ & $0.24-0.28$ \\
& Magnesium diboride $\left(\mathrm{MgB}_{2}\right)[32]$ & $0.33-0.42$ \\
& Bismuth telluride $\left(\mathrm{Bi}_{2} \mathrm{Te}_{3}\right)[45]$ & $0.31-1.38$ \\
VIS-NIR & Bismuth selenide $\left(\mathrm{Bi}_{2} \mathrm{Se}_{3}\right)[45]$ & $0.709-1.180$ \\
& Ruthenates $\left(\mathrm{Sr}_{2} \mathrm{RuO}_{4}, \mathrm{Sr}_{3} \mathrm{Ru}_{2} \mathrm{O}_{7}\right)[33]$ & 1.49 \\
& Hexagonal boron nitride $(\mathrm{hBN})[31]$ & $6.2-7.3,12.1-13.2$ \\
& $\alpha$ - $\mathrm{MoO}_{3}[46,47]$ & $9.9-18.3$ \\
& $\alpha$ - $\mathrm{V}_{2} \mathrm{O}_{5}[48]$ & $9.6-19.7$ \\
& Sapphire $\left(\mathrm{Al} \mathrm{O}_{3}\right)[36]$ & 20 \\
& Bismuth $(\mathrm{Bi})[36]$ & $53.7-63.2$ \\
Calcite $\left(\mathrm{CaCO}_{3}\right)[44]$ & $6.45-7.09,13.99-14.04$ \\
\hline
\end{tabular}

anisotropic crystal structures are arrayed in layers, and the natural hyperbolicity results from the two-dimensional conductivity of the parallel atomic plane. The operating wavelength of these materials is intrinsically bounded by their natural crystal structure and can only be slightly tuned by environmental factors. Various naturally occurring hyperbolic materials are summarized in Table 1 according to their operating wavelengths.

However, artificially structured HMMs may be beneficial due to the fact that their optical properties can be easily adjusted by changing the constituent meta-atoms. HMMs have both metallic and dielectric properties depending on the direction, so the most straightforward way to realize HMMs is to design structures composed of metals and dielectrics. So far, two structures have been typically used for bulk HMMs: alternating multilayers of sub-wavelength thickness metal-dielectrics (multilayer HMMs) [49, 50], and metallic NWs embedded in a dielectric host medium (NW HMMs) $[26,51]$. The realization of HMMs using these structures relies on effective medium theory
(EMT) [52], which describes the macroscopic optical responses of composite materials using homogenized effective parameters. When the size of the constituent materials is sufficiently small, the homogenization is valid and a hyperbolic effective permittivity tensor can be achieved.

Multilayer HMMs are the simplest structures, in which the motion of free electrons is restricted to directions parallel to the interfaces between layers. In sub-wavelength thickness multilayer HMMs, the effective permittivity components parallel $\varepsilon_{\|}$and perpendicular $\varepsilon_{\perp}$ to the OA can be approximated as [34]

$$
\begin{aligned}
\varepsilon_{\|} & =\varepsilon_{z z}=\left(\frac{f}{\varepsilon_{m}}+\frac{1-f}{\varepsilon_{d}}\right)^{-1}, \\
\varepsilon_{\perp} & =\varepsilon_{x x, y y}=f \varepsilon_{m}+(1-f) \varepsilon_{d},
\end{aligned}
$$

where $\varepsilon_{m}$ and $\varepsilon_{d}$ are the permittivity of the metal and dielectric materials, respectively, and $f=\frac{t_{m}}{t_{m}+t_{d}}$ is the volume fraction of metal and dielectric with thicknesses of $t_{m}$ and $t_{d}$, respectively. The effective permittivity can be customized by the choice of materials and $f$. The tuning of these two parameters can yield both Type-I and Type-II multilayer HMMs, as well as effective dielectric or metallic properties. Multilayer HMMs fabricated with evaporation or sputtering deposition have generally been used to create horizontal layers. However, to exhibit Type-I HMMs, this structure requires an optical resonance, which results in a narrow working wavelength and high resistive losses. Recently, vertically-alternating multilayer HMMs have been proposed to circumvent these optical resonances and achieve Type-II HMMs [27, 53, 54].

The use of NW HMMs is another approach to achieve HMMs, where metallic NWs are embedded in a dielectric host medium. In NW HMMs that have sub-wavelength diameters and lattice periods, the effective 
permittivity tensor components parallel $\varepsilon_{\|}$and perpendicular $\varepsilon_{\perp}$ to the OA are [34]

$$
\begin{aligned}
& \varepsilon_{\|}=\varepsilon_{z z}=f \varepsilon_{m}+(1-f) \varepsilon_{d}, \\
& \varepsilon_{\perp}=\varepsilon_{x x, y y}=\frac{\varepsilon_{d}\left[(1+f) \varepsilon_{m}+(1-f) \varepsilon_{d}\right]}{(1+f) \varepsilon_{d}+(1-f) \varepsilon_{m}},
\end{aligned}
$$

where $\varepsilon_{m}$ and $\varepsilon_{d}$ are the permittivity of the metal wire and dielectric host, respectively, and $f$ is the volume fraction of the metal. The real part of the metal permittivity is negative at frequencies below the plasma frequency, so the NW structures can easily achieve Type-I HMMs without the requirement of an optical resonance. Consequently, they can operate at relatively low loss and high transmission over a large bandwidth. Due to the longwavelength limit condition, structures should have a high aspect ratio with an extremely small diameter compared to the slab thickness. Those structures with high aspect ratio have been mainly fabricated using electrochemical deposition methods in which metals such as silver $(\mathrm{Ag})$ and gold $(\mathrm{Au})[21,55]$ are grown inside an anode alumina template. The electrochemical deposition method allows for efficient large-area fabrication, but limits the choice of materials that can be used.

\subsection{D planar hyperbolic materials}

The unique hyperbolic IFC of bulk HMMs have demonstrated the capability to control light in unconventional ways, but they generally have high propagation losses and demanding fabrication requirements. Accordingly, attempts to compress bulk HMMs to 2D surfaces have been made to alleviate this problem. Metasurfaces are 2D planar devices that can manipulate light with the advantages of having a compact form factor and lower propagation losses than 3D structures [17, 56, 57]. In addition, the simplified fabrication and integration processes of metasurfaces facilitate the compatibility of integration with other photonic devices. Metallic metasurfaces exploit the control of the propagation of surface plasmon polaritons (SPPs) along the metasurfaces. For these ultra-thin metasurfaces, the effective surface conductivity tensors, which are analogous to the effective dielectric tensor, Eq. (5) are used to describe the homogenized medium, as

$$
\sigma=\left(\begin{array}{cc}
\sigma_{x x} & 0 \\
0 & \sigma_{y y}
\end{array}\right)
$$

where $\sigma_{x x}$, and $\sigma_{y y}$ are the $x$ and $y$ components of the surface conductivity. Metasurfaces can support both TE and TM modes, but the confinement of the TE mode is not as strong as the TM mode. Therefore, we only consider TM modes confined to metasurfaces. The dispersion relation of TM modes in metasurfaces is given as $[58,59]$

$$
\eta_{0}^{2}\left(k_{x}^{2} \sigma_{x x}+k_{y}^{2} \sigma_{y y}\right)^{2}\left(k_{x}^{2}+k_{y}^{2}-k_{0}^{2}\right)-4 k_{0}^{2}\left(k_{x}^{2}+k_{y}^{2}\right)^{2}=0,
$$

where $\eta_{0}$ is the impedance of free-space. HMSs are extremely anisotropic metasurfaces that exhibit a hyperbolic dispersion of in-plane SPPs [58, 60, 61]. That is, the sign of the imaginary parts of the effective surface conductivity components are different to each other. Consequently, HMSs exhibit both metallic (inductive, $\operatorname{Im}(\sigma)>0$ ) and dielectric (capacitive, $\operatorname{Im}(\sigma)<0$ ) properties depending on the direction of surface conductivity. Due to the unique dispersion relation of in-plane SPPs, HMSs can support extremely large wave vectors, and have a large photonic density of states, so many interesting phenomena can be achieved [61-63]. In practice, HMSs have been realized with either metallic gratings [61] or natural 2D hyperbolic materials such as graphene $[58,64,65]$ and hBN $[66,67]$ at high frequencies. However, at low frequencies, noble metals are regarded as perfect electric conductors, in which SPPs become weakly confined. Instead, structured metal surfaces can confine surface waves called spoof SPPs [68, 69], where the structured surfaces allow the support of cut-off frequency modes and sub-wavelength confinement at low frequencies. Spoof SPPs can mimic the novel properties of SPPs, and the dispersion of spoof SPPs can be simply tailored by adjusting the geometrical parameters of the structured surface. Therefore, HMSs that support the propagation of spoof SPPs in the microwave regime have been demonstrated using structured metal resonator structures [70,71].

\section{Applications of hyperbolic metamaterials 3.1 High-resolution optical imaging and nanoscale lithography}

The diffraction limit prevents conventional microscopes from observing sub-wavelength features [74-76]. This limit is a result of the depletion of evanescent waves at the image plane in the far-field. The light scattered from an object is composed of various spatial frequency components. The low- $k$ waves contain information about the large geometric features of the object, and are able to propagate in a vacuum. On the contrary, the dispersion relation in a vacuum ensures that the longitudinal component for wave with transverse wave vector higher than $k_{0}$ (high- $k$ wave) necessarily becomes imaginary. Consequently, the waves that encode the sub-wavelength features decay exponentially and cannot reach the image plane (inset (i) in Fig. 2a).

The hyperbolic dispersion in HMMs supports the propagation of the high- $k$ waves, therefore showing the potential for super-resolution imaging [21, 34]. The unbounded IFC of HMMs allows arbitrarily large 
a

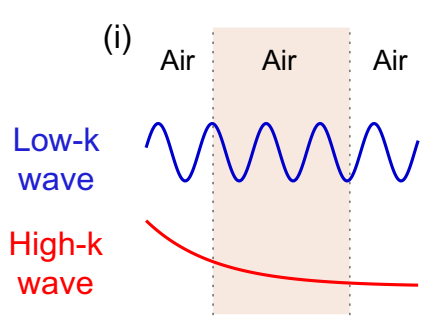

b

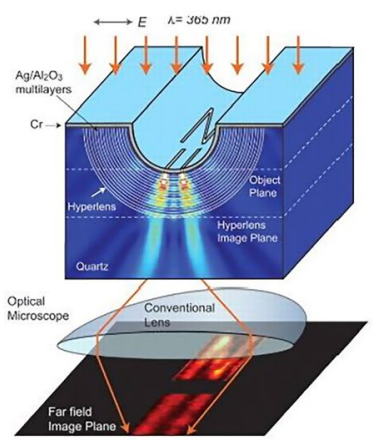

C

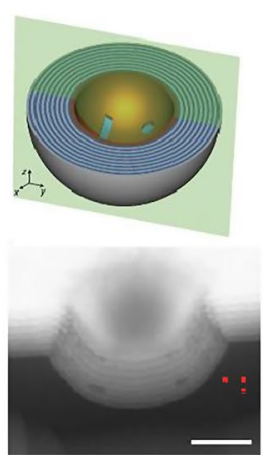

(ii)

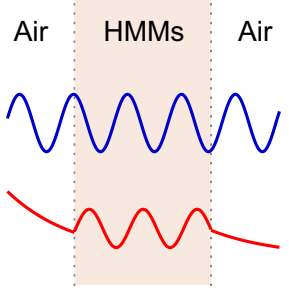

d
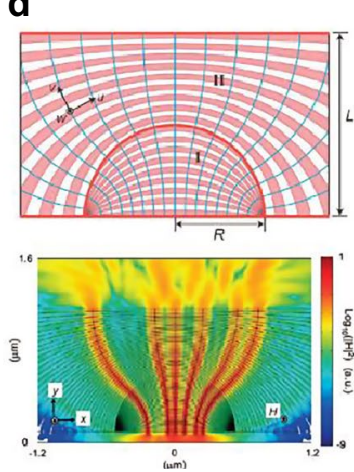

(iii) Air Hyperlens Air
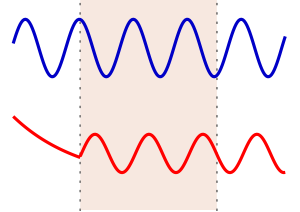

e

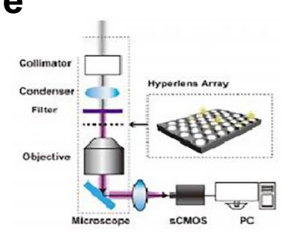

Fig. 2 Sub-wavelength imaging using HMMs. a Schematics of light propagation in (i) air, (ii) HMMs, and (iii) hyperlens. b Cylindrical hyperlens composed of alternating layers of silver ( $\mathrm{Ag}$ ) and silicon dioxide $\left(\mathrm{SiO}_{2}\right)$. c A schematic and scanning electron microscope (SEM) image of spherical hyperlens made of $\mathrm{Ag}$ and titanium dioxide $\left(\mathrm{TiO}_{2}\right)$. $\mathbf{d}$ A planar hyperlens designed by transformation optics and its simulation result. $\mathbf{e}$ Sub-wavelength imaging system combined with array type hyperlens and SEM images of the fabricated wafer-scale hyperlens. b Reproduced with permission from ref. [24], copyright 2008, AAAS. c Adapted with permission from ref. [25], copyright 2010, NPG. d Adapted with permission from ref. [72], copyright 2008, ACS. e Adapted with permission from ref. [73], copyright 2017, ACS

transverse wave vectors with real values of longitudinal wave vectors. The first theoretical concepts of superresolution imaging have been demonstrated using several HMMs [77-79], but the waves become evanescent again as the waves exit the HMMs (inset (ii) in Fig. 2a). A hyperlens is a device designed to overcome this limitation [24, 25, 80]. Hyperlenses made of HMMs utilize their geometrically-curved shapes to magnify the subwavelength information as well as supporting high- $k$ waves (inset (iii) in Fig. 2a). When the wave propagates in the radial direction of a hyperlens, the transverse wave vectors are compressed in accordance with the law of conservation of angular momentum; i.e., the image is gradually magnified as it is transferred along the radial direction. The magnification ratio of the hyperlens can be estimated from the ratio of the inner and outer radii. Therefore, evanescent waves can be converted to propagating waves by carefully choosing a radius ratio that sufficiently compresses the transverse wave vectors. The converted waves can then be projected onto the image plane in the far-field, and thereby achieve super-resolution. Hyperlenses can be readily integrated into conventional optical systems [73, 81] proving their promise for practical applications.
Super-resolution imaging using a hyperlens was first demonstrated in the ultraviolet (UV) regime [24]. A cylindrical hyperlens composed of alternating concentric multilayers of $\mathrm{Ag}$ and $\mathrm{Al}_{2} \mathrm{O}_{3}$ (Fig. 2b) was used to achieve a resolution down to $\lambda_{0} / 3$, where $\lambda_{0}$ is the wavelength of light. However, the cylindrical shape restricts its magnification to only one direction, so the geometrical shape was then extended to a spherical shape to achieve full-lateral sub-wavelength imaging (Fig. 2c) [25]. Still, the curved inner surfaces of the cylindrical or spherical hyperlens makes sample placement difficult and hinders their practical applications. As alternatives, planar hyperlenses [72, 82-84] were developed (Fig. 2d). Planar hyperlenses can be designed using transformation optics [82], where the light path is deliberately modulated to magnify the subwavelength features.

Recent studies on hyperlenses have sought to address some of the practical limitations [73, 85]. Hyperlenses are generally inefficient due to high reflection and absorption losses. In particular, the efficiency decreases as the thickness of the hyperlens is increased, which in turn limits the achievable magnification. One factor responsible for the low efficiency is the huge impedance mismatch at the surface of a hyperlens, so to reduce the high reflection, 
impedance-matched hyperlenses have been proposed [86]. A non-resonant hyperlens with a radial (fan-shaped) configuration has been demonstrated to reduce the absorption caused by resonances [85]. The radially alternating metal-dielectric multilayer allows the realization of a hyperbolic dispersion in a non-resonant way, and hence low losses in the visible range. The correct selection of materials can also avoid the intrinsic losses of metals. Non-metallic materials of transparent conducting oxides [87] have been used to replace lossy metals. To alleviate the sample-positioning problem, a wafer-scale hyperlens device has been proposed (Fig. 2e) [73]. A scalable hyperlens array was fabricated on a 4-in. wafer, and real-time imaging of biological systems using the hyperlens array was demonstrated as a practical application. The characteristic of HMM to support sub-diffractionlimited optical patterns has recently been exploited to improve the resolution of conventional microscopy by combining HMMs with conventional structured illumination microscopy [88-90]. HMMs are used to generate sub-diffraction-limited structured light patterns, and the scattering fields are collected in the far-field to improve spatial resolution down to $\sim 40 \mathrm{~nm}[90]$.

Sub-diffractional focusing and imaging using natural hyperbolic materials have also been demonstrated to avoid intrinsically high optical losses caused by metals in HMMs [31, 67, 91-95]. In contrast to HMMs, the upper limit of the propagating wave vector of natural hyperbolic materials can be greatly extended due to their homogenous nature, and it is only limited by the crystal lattice periodicity [96]. In addition, the long scattering lifetime properties of phonon polaritons supported by natural hyperbolic materials make them attractive candidates for imaging. Therefore, near-field focusing and imaging have been actively demonstrated using natural hyperbolic materials such as hBN [31, 67, 91, 92, 97], graphene [94, 95], and $\alpha-\mathrm{MoO}_{3}$ [93]. Recently, by exploiting ultra-low loss monoisotopic materials of $\mathrm{h}^{11} \mathrm{BN}$, hyperlens device with significantly improved spatial resolution down to $\lambda / 154$ has been demonstrated [97].

The unique properties of HMMs beyond the diffraction limit are also helpful in generating deep sub-wavelength patterns in lithography [98-101]. The reciprocity theorem suggests that a hyperlens can also be used to reduce diffraction-limited patterns to sub-diffraction patterns. Sub-wavelength photolithography using hyperlenses was therefore demonstrated by simply reversing the direction of the incident light $[98,100]$. As the light travels along the radial direction to the inner radius, the transverse wave vectors are gradually increased, so the pattern size decreases (Fig. 3a). The first experimental demonstration of a de-magnifying hyperlens using an $\mathrm{Ag}-\mathrm{TiO}_{2}$ multilayered structure [53]. A pattern with line-width of 300 $\mathrm{nm}$ inscribed on the outer hyperlens surface was reduced to $170 \mathrm{~nm}$ (a de-magnification ratio of 1.8). Recently,

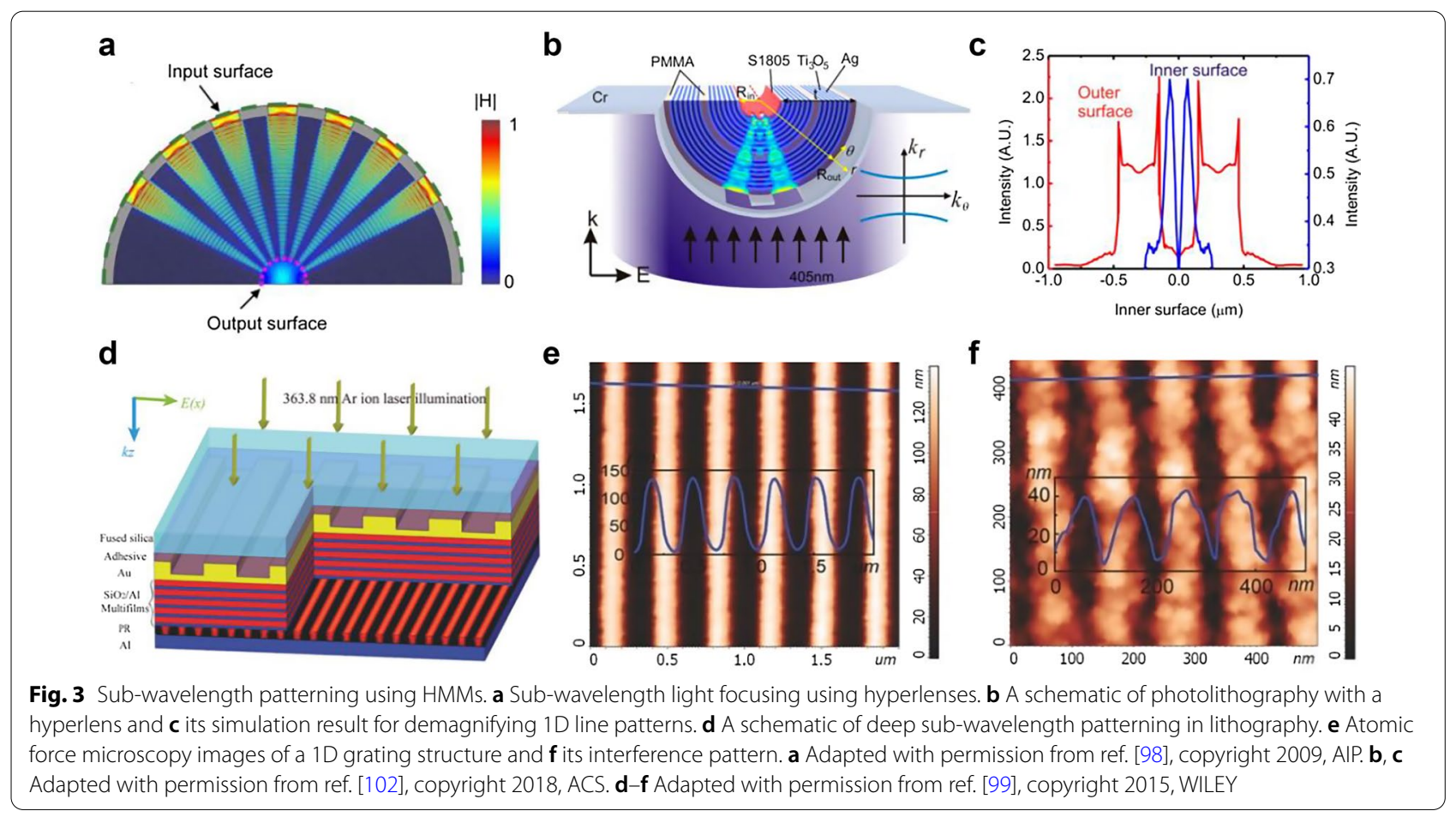


a de-magnification ratio of 3.75 was achieved using a hyperlens with a high radius ratio (Fig. 3b, c) [102].

Other approaches that use HMMs to obtain high-resolution patterns have been used in interference lithography $[101,103]$. Laser interference lithography is an inexpensive large-area nanofabrication method that utilizes the periodicity of the interference pattern between light waves [104]. Therefore, the resolvable pattern size is again restricted by the diffraction limit, so methods to decrease the operating wavelength are being sought $[105,106]$. HMMs can support high- $k$ waves, and therefore have the ability to generate sub-wavelength patterns [99, 101, 107]. Numerical simulations suggested that a conventional chromium grating with $320 \mathrm{~nm}$ period can generate a $40 \mathrm{~nm}$-sized sub-wavelength pattern [101]. Subsequent rigorous analysis of the mechanism and properties of the SPPs in HMMs have extended the understanding of the formation of sub-wavelength interference patterns [107]. Sub-wavelength interference lithography using HMMs has been experimentally demonstrated using $\mathrm{Al}_{-} \mathrm{SiO}_{2}$ multilayers (Fig. 3d) [99]. The mutual coupling of SPPs between adjacent metal-dielectric interfaces results in bulk plasmon polariton modes, which generate large-area interference patterns. An Au grating with a period of $360 \mathrm{~nm}$ (Fig. 3e) was used to generate uniform interference patterns with a linewidth of $45 \mathrm{~nm}$ (Fig. 3f) over a large area of $20 \mathrm{~mm} \times 20 \mathrm{~mm}$. Recently, the use of Type-II epsilon-near-zero HMMs for high transmission has achieved high fidelity deep subwavelength lithography [108].

\subsection{Light propagation and manipulation using HMMs}

The hyperbolic dispersion of HMMs provides a innovative method of manipulating the propagation of light. Among the various light-propagation phenomena that have been achieved using HMMs, negative refraction has been widely discussed. Various metamaterials such as split-ring resonators [112], multilayered fishnet [113], chiral [40, 114, 115], and asymmetric nanoring [116] structures have been employed to achieve negative refraction. HMMs lead to negative refraction under TM polarized light which is affected by $\varepsilon$. Therefore, negative refraction using HMMs can be realized by adjusting $\varepsilon$ even if neither $\varepsilon$ nor $\mu$ have negative values that are needed for a negative $n$. The principle of negative refraction using HMMs can be described using the IFC (Fig. 4a). In conventional isotropic media such as air, the wave vector $k$ and Poynting vector $\mathbf{S}$ of TM mode light are parallel. However, if TM mode light propagates along the interface between a HMM and air, $k$ and $\mathbf{S}$ are no longer parallel. If we consider the $x$ - $z$ plane between a Type-II HMM and air, negative refraction can be observed because the tangential components of $k$ and
S point in opposite directions [80, 117]. This negative refraction implies energy-negative refraction only when light passes through the HMM in an isotropic medium, which differs from the case of an isotropic left-handed metamaterial, in which the phase and energy are negative simultaneously. The direction of propagation and the refracted light is anti-parallel $[118,119]$.

Various investigations of negative refraction have been performed using HMMs and HMSs at different wavelengths $[26,61,109-111]$. At visible wavelengths, negative refraction has been experimentally demonstrated using HMMs composed of sub-wavelength $\mathrm{Ag} \mathrm{NW}$ structures (Fig. 4b) [26]. The fabricated bulk Ag NW HMM has opposite signs of $\varepsilon$ along the perpendicular and parallel directions of the structures, producing a hyperbolic dispersion to achieve low-loss wide-angle negative refraction at wavelengths from 660 to $780 \mathrm{~nm}$. Negative refraction of SPPs has also been numerically demonstrated using HMSs [109]. The simulations demonstrated that the SPPs travel from the metal surface to the HMS, and negative refraction occurs at the interface. A simple diffraction-grating structure was used to control the dispersion of the SPPs, and broadband wide-angle negative refraction was demonstrated. The dispersion relation of the grating coupled metasurface represents a hyperbolic dispersion that is flat around $\lambda=$ $543 \mathrm{~nm}$ (Fig. 4c). At this wavelength, the SPPs propagate without diffraction with a group velocity normal to the IFC, so negative refraction of SPPs is observed (Fig. 4d). Negative refraction from metasurfaces has been demonstrated experimentally [61]. Metasurfaces composed of single-crystalline Ag films showed a hyperbolic dispersion below the critical wavelength of $\lambda=540 \mathrm{~nm}$ (Fig. 4e). At $\lambda>540 \mathrm{~nm}$, the single-crystalline Ag film has an elliptical dispersion. SPPs that propagate along the metal film meet the interface between metal and singlecrystalline Ag film, and are refracted. The propagation of SPPs changes to negative refraction only at the hyperbolic wavelength region below $\lambda=540 \mathrm{~nm}$, this result shows that negative refraction in HMSs is dependent on the wavelength of the incident light (Fig. 4f).

In the near-infrared region, an experimental demonstration of negative refraction using HMM with alternating multilayers of zinc oxide $(\mathrm{ZnO})$ and aluminum-doped zinc oxide (AZO) has been reported [110]. Hyperbolic dispersion was achieved at $1.84 \mu \mathrm{m}$ $\leq \lambda \leq 2.4 \mu \mathrm{m}$ (Fig. $4 \mathrm{~g}$ ). Measurements of the blocking of propagating light when negative refraction occurred show a clear dip in the transmittance spectrum to prove the existance of negative refraction (Fig. 4h). In the far-infrared region, wide-angle negative refraction has been achieved using a multilayer of thick semiconductor layers of doped indium gallium arsenide 


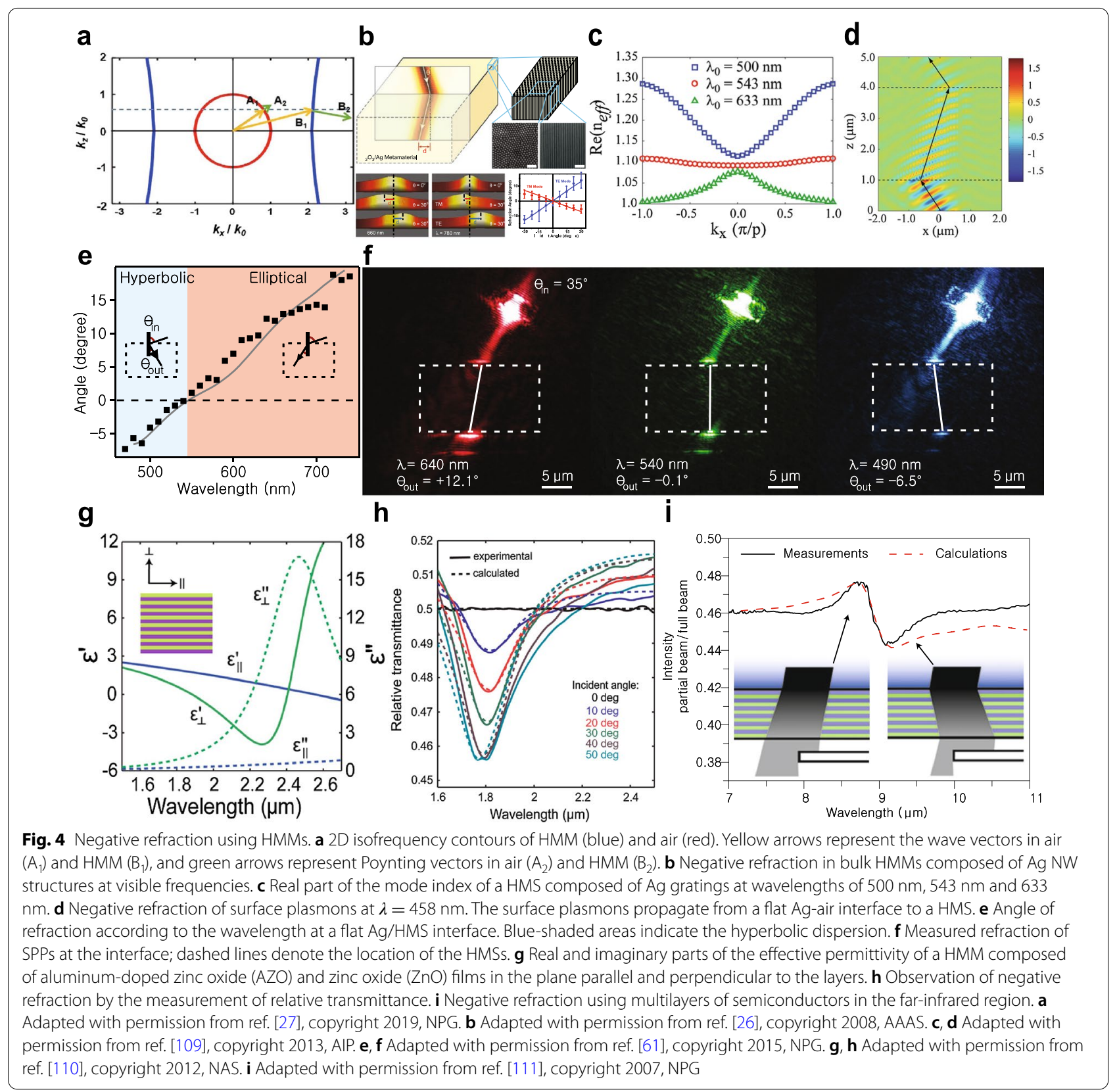

(InGaAs) and intrinsic AllnAs that has low-loss properties (Fig. 4i) [111]. Negative refraction in the terahertz/gigahertz regions has also been achieved using natural hyperbolic materials, which will be introduced in more detail in Sect. 3.5. All-angle highly-squeezed polariton negative refraction was achieved using HMSs composed of anisotropic or nanostructured 2D materials over a broadband wavelength region. The working bandwidth can also be varied by tuning the chemical potential of the materials [120]. Moreover, experiments have demonstrated HMSs that have an anisotropic magnetic sheet conductivity supporting in-plane high$k$ designer polaritons. This phenomenon overcomes the difficulty of practical realization despite various theoretical proposals of negative in-plane refraction [121].

Negative refraction through multilayered bulk HMMs has been typically achieved using horizontally-stacked multilayered HMMs (hHMMs). A broad range of working wavelengths $\triangle \lambda_{\mathrm{w}}$ and low-loss are desirable characteristics, so hHMMs are limited by their use of narrows resonances $\triangle \lambda_{\mathrm{w}}$ and high losses. To alleviate these drawbacks, recently, HMMs composed of 
a

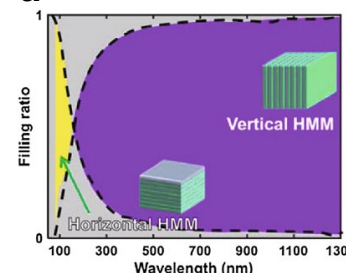

C b

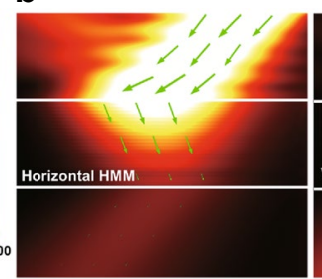

0

$-1$

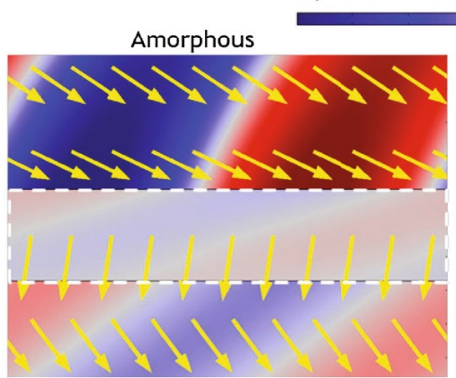

Air

1 [a.u.]

Crystalline

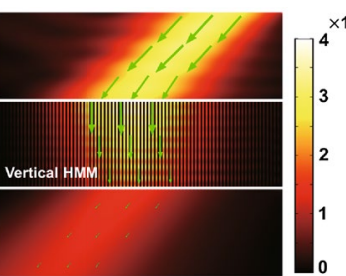

d
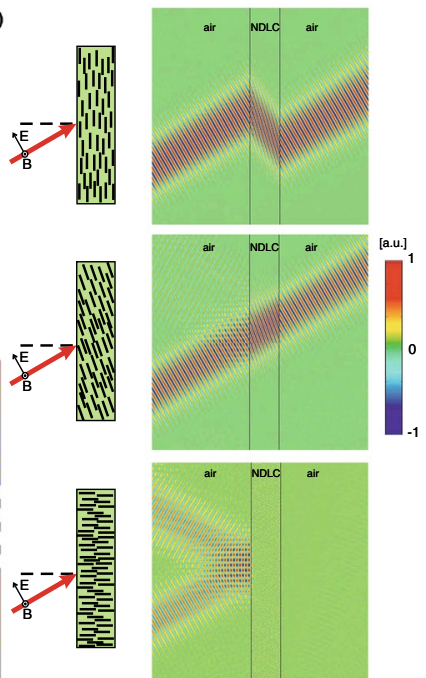

Fig. 5 Negative refraction using vertical and switchable HMMs. a Effective permittivity in horizontally-stacked multilayered HMMs (hHMMs) and vertically-stacked multilayered HMM (vHMMs), and $\mathbf{b}$ intensity profiles in $\mathrm{hHMMs}_{\mathrm{H}}$ and $\mathrm{vHMMs}$ composed of aluminum (Al) and SiO 2 . Green arrows: Poynting vectors. c Switchable refraction between negative to positive using layers of phase change materials (PCM). Dashed lines: HMM; arrows: power flow for transverse magnetic (TM) polarized light. d Switchable refraction from negative to positive and reflection, using liquid crystal HMMs. a, b Adapted with permission from ref. [27], copyright 2019, NPG. c Adapted with permission from ref. [122], copyright 2018, WILEY. d Adapted with permission from ref. [123], copyright 2014, OSA

vertically-stacked multilayered HMMs (vHMMs) have been demonstrated to realize broadband negative refraction [27]. The calculated effective permittivity of aluminum $(\mathrm{Al})$ and $\mathrm{SiO}_{2}$ multilayer structures show broader $\triangle \lambda_{\mathrm{w}}$ in vHMMs than in hHMMs in the visible range (Fig. 5a). The negative refraction of vHMMs is not dependent on resonances, so they can achieve wide $\triangle \lambda_{\mathrm{w}}$ and avoid losses that originate from resonances [124]. Therefore, the transmission in vHMMs can be higher than in hHMMs. Numerical demonstrations using the finite-element method verified the clear negative refraction from vHMMs with higher transmittance than hHMMs (Fig. 5b). A numerical study of the critical layer thickness of vHMMs in the visible region has been reported [125]. A variety of vHMMs composed of various materials have been numerically investigated to determine the critical layer thickness of the potential for negative refraction utilization. Recently, a vHMM composed of $\mathrm{Au}$ and copolymer resist (EL8) has been fabricated through two-step focused ion beam milling. This vHMM experimentally demonstrates broadband negative refraction between $450 \mathrm{~nm}$ and $550 \mathrm{~nm}$ in the visible region [126].

Phase change materials and liquid crystals have also been applied to HMMs to achieve active control of the negative refraction. One such switchable HMM used a chalcogenide glass alloy of $\mathrm{Ge}_{2} \mathrm{Sb}_{2} \mathrm{Te}_{5}$ [122], which undergoes a phase change from amorphous to crystalline. By using a $\mathrm{Ge}_{2} \mathrm{Sb}_{2} \mathrm{Te}_{5}$ nanocomposite as one of the layers in a multilayer structure HMM, the phase change yields a switchable Type-I hyperbolic dispersion and subsequently the negative refraction changes from the visible to near-infrared region (Fig. 5c).

Nanosphere-dispersed liquid crystals (NDLCs) have also been applied to achieve switchable HMMs in the near-infrared region [123]. Appropriate application of an external electric field was shown to produce a switching mechanism between normal refraction, negative refraction, and reflection of TM mode light (Fig. 5d). Negative refraction using 3D assembled nanorod arrays [127] and using 2D materials such as graphene, boron nitride, and organic materials has also been investigated, the latter of which will be introduced in detail in the following section [120, 128-130].

In addition to negative refraction, the spin Hall effect of light (SHEL) [133-137] is another interesting application of HMMs. SHEL refers to a transverse and spindependent displacement of light. In the conventional spin Hall effect, spin-up and spin-down electrons are deflected in opposite directions, whereas in the SHEL, refracted or reflected light at an optical interface undergoes spin-dependent splitting (Fig. 6a). The shift of the refracted light can be calculated from the incident angle and Fresnel coefficients as $\delta_{H}=\lambda \cot \theta_{i} \operatorname{Re}\left(1-t_{s} / t_{p}\right) / 2 \pi$ 


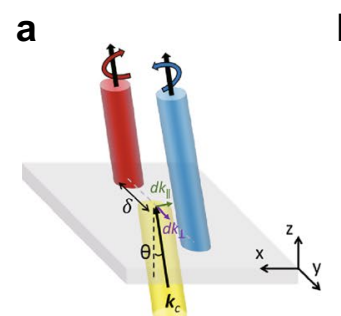

d

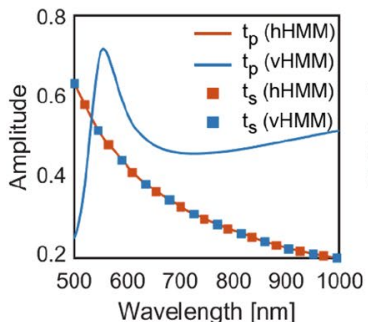

b

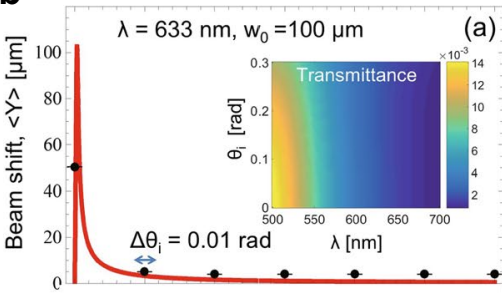

e

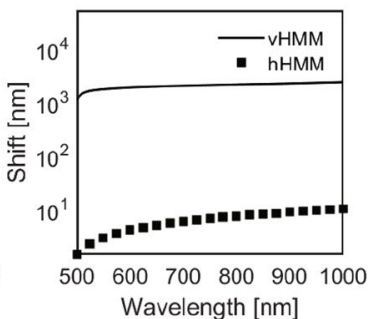

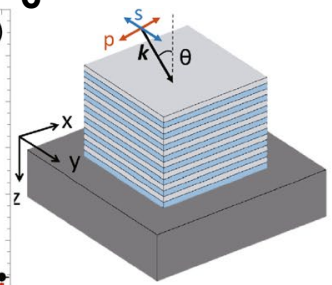

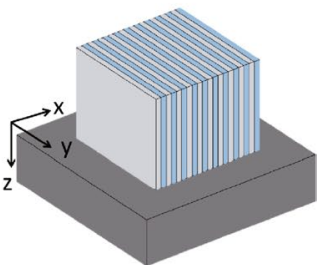

g
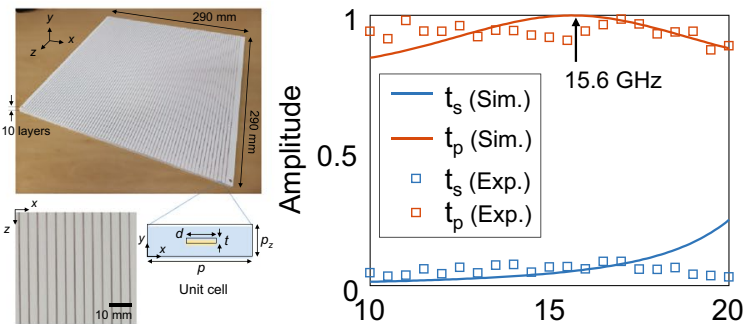

Fig. 6 Spin Hall effect of light using HMMs. a Schematic of spin Hall effect of light (SHEL). b Numerical and experimental demonstration of SHEL from $\mathrm{hHMM}$ consisting of aluminum oxide $\left(\mathrm{Al}_{2} \mathrm{O}_{3}\right)$ and gold $(\mathrm{Au})$ at the visible frequency of $\lambda=633 \mathrm{~nm}$. c Schematics of hHMM and vHMM. $\mathbf{d}$ Amplitude of transmission coefficients and $\mathbf{e}$ calculated shift of light when the incident angle of $5^{\circ}$. $\mathbf{f} \mathrm{A}$ photograph and schematic of wire based HMM having near-unity transmission for one polarization. $\mathbf{g}$ Numerical and experimental demonstration of near-unity efficiency SHEL at $15.6 \mathrm{GHz}$. a, c-e Adapted with permission from ref. [54], copyright 2019, ACS. b Adapted with permission from ref. [131], copyright 2018, OSA. f-g Adapted with permission from ref. [132], copyright 2020, WILEY

for horizontally polarized incidence and as $\delta_{V}=\lambda \cot \theta_{i} \operatorname{Re}\left(1-t_{p} / t_{s}\right) / 2 \pi$ for vertically polarized incidence [135]. The shift is generally much smaller than the wavelength, so effort have been devoted to increasing the shift using metamaterials and metasurfaces [132, $138,139]$. A straightforward way to increase the shift is to design a medium that has polarization-dependent transmission or reflection characteristics. Hyperbolic metamaterials offer a natural candidate to increase the shift from the SHEL.

Amplification of the SHEL using HMMs has been proposed theoretically using planar [140, 141] and curved multilayers [142], while experimental demonstration has been achieved in the visible range using a horizontallystacked multilayer [131]. A large incident-angle-sensitive SHEL shift that reached $165 \mu \mathrm{m}$ was also achieved experimentally (Fig. 6b). The SHEL may be further amplified by several orders of magnitude through the use of vertically-stacked HMMs instead of horizontally-stacked HMMs [54]. vHMMs have a different OA configuration than horizontal HMMs (Fig. 6c), which results in a large discrepancy between the transmission of s- and p-polarized light (Fig. 6d). A numerical demonstration showed an increase in the shift of several orders of magnitude, and an experimental demonstration using a vHMM with an $\mathrm{Au}$ nanograting verified a micrometer scale increase in transverse shift (Fig. 6e).
The analytic formula of the shift implies that the shift diverges as the Fresnel coefficient in the denominator approaches zero. However, the efficiency of the SHEL is defined as the intensity of the spin-separated beam divided by the intensity of the incident light [132] which decreases quickly due to the relationship with the square of the Fresnel coefficient in the denominator. Thus, many previous attempts to increase the SHEL have been marred with low efficiency. A HMM composed of an array of metallic wires in a dielectric host has been suggested to achieve a large SHEL with near-unity efficiency (Fig. 6f) [132]. Because of the in-plane anisotropy, the wire-based HMM structure shows near-unity transmission for one polarization but extremely low transmission for the other (Fig. 6g). The numerically and experimentally-obtained shifts and efficiencies agreed well and proved a large shift that simultaneously reached 1.57 wavelengths and near $100 \%$ efficiency in the microwave regime.

\subsection{Spontaneous emission engineering}

After the discovery that spontaneous emission can be increased using a resonant cavity, research in photonic and plasmonic fields has verified the increased spontaneous emission rate through the engineering of the local density of states (LDOS) near cavities or nanostructures [143]. HMMs have shown the potential as an alternative 
to resonant cavities. The resonance in cavities hinders the development of broadband spontaneous emission and highly-confined electromagnetic fields. In contrast, the HMMs can be a promising solution for enhancing the spontaneous emission over the broad bandwidth. From Fermi's golden rule, the radiative spontaneous emission rate can be defined as $[144,145]$

$$
\Gamma=\frac{\pi \omega_{0}}{3 \hbar \varepsilon_{0}}|\mathbf{p}|^{2} \rho\left(r_{0}, \omega_{0}\right)
$$

where $\mathbf{p}$ is a matrix that expresses the transition dipole moment of energy states, $\rho\left(r_{0}, \omega_{0}\right)$ is the LDOS, $r_{0}$ is the position of the emitter, and $\hbar \varepsilon_{0}$ is the photon energy. Equation (7) demonstrates that modification of the electromagnetic environment around the emitters, especially by an adjustment of the LDOS, can increase the spontaneous emission rate involving any factors related to the nature of emitters such as transition dipole moment, chemical structure, and energy levels in the transition. In this respect, the HMMs that support the open IFCs and thus diverging LDOS substantially enhance the spontaneous emission rate. Thus, research on HMMs has improved the manipulation of spontaneous emission which can be characterized from the measured emission spectrum and lifetime of the emitters.

HMMs have been studied the characteristics of light emitted from a dye or dye-containing layer on a NW or metal-dielectric multilayer-based structure. The effects of HMMs on the increase of spontaneous emission have been studied theoretically and experimentally $[28,29$, 149, 151-156]. The enhanced spontaneous emission rate near the HMMs is characterized by the Purcell factor, defined as the total emitted power of the source near the HMMs by that at the air. Furthermore, to maximize the effect of spontaneous emission due to the confinement of higher wave vectors using HMMs, research has been actively conducted to examine the effect of placing the emitter directly inside the $\operatorname{HMM}[147,157,158]$. Although many researchers have used multilayer HMMs to manipulate the spontaneous emission of dyes or emitters, the focus has only been on observing the Purcell factor of emitters located near HMMs. Most of these studies could not overcome the limitation of multilayered thin-film-type HMMs that have non-radiative properties. Trials to increase the radiative emission efficiency from HMMs are required to enhance the spontaneous emission and yield high enough Purcell effect that can be detected even in the far-field. Therefore, outcoupling techniques have been applied to HMMs to effectively control and improve the LDOS and radiative emission [146-148, 157, 159-168]. Recently, photoluminescence has been increased by using HMMs composed of films of deposited perovskites [169] and HMMs composed of coupled perovskite nanocrystals [170].

The advantages of HMMs can be explained by comparing the Purcell factors of single metal layers and HMMs (Fig. 7a, b) [146]. The Purcell factors are calculated for the UV to visible range for each structure, with an emitter 10 $\mathrm{nm}$ away from the single Ag film and Ag-Si multilayer. A bulk single Ag layer has a high Purcell factor within a narrow bandwidth at a surface plasmon resonance in the UV region (Fig. 7a). On the other hand, increase the number of alternating layers results in interface plasmon polaritons that have shifted resonance frequency. These multiple bands of plasmon polaritons broaden the bandwidth of the enhanced Purcell factor [34]. Thus, the HMM composed of a Ag-Si multilayer has a high Purcell factor over a broad range of visible frequencies (Fig. 7b). By adjusting the filling ratio of the metal in HMMs, the position of the peak of the Purcell factor can be freely controlled within the target wavelength range (Fig. 7c). In addition, by exploiting the outcoupling effect of a grating or nanopatterns, radiative emission can be increased compared to flat multilayer HMMs. The Purcell factor and radiative enhancement at the emission wavelength can be observed through the comparison of uniform HMMs and nanopatterned multilayer HMMs fabricated by focused ion beam milling. Both uniform and nanopatterned HMMs have a high Purcell factor (Fig. 7d), but the calculated radiative emission results show remarkable differences (Fig. 7e). The radiative enhancement on the nanopatterned HMM at the peak wavelength is almost double; this change increases the external quantum efficiency of the emitters. HMMs can also be outcoupled using bullseye gratings [157], nanoantennas (Fig. 7f) [147], 2D grating patterns, and rolled-up structures (Fig. 7g) [148] for the enhancement of the directional emission of dipoles.

Emission can also be manipulated by controlling the topological transitions in HMMs [149, 171]. A transition in the topology of the IFC can be induced by a shift of the wavelength, and as for the $\mathrm{HMM}$ composed of $\mathrm{Ag}-\mathrm{TiO}_{2}$, the IFC may change from closed (elliptical) to open (hyperbolic dispersion) at a specific wavelength (Fig. 7h).

Phase-change materials (PCMs) have been used to develop tunable HMMs to control the spontaneous emission. A topological transition from an elliptical to a hyperbolic dispersion can be achieved for tunable HMMs by incorporating a PCM. The LDOS near a HMM can change according to the phase of the PCM, so the emission is also affected. A tunable HMM composed of titanium nitride (TiN) and the PCM, antimony sulfide $\left(\mathrm{Sb}_{2} \mathrm{~S}_{3}\right)$, demonstrated a considerable tunability of the Purcell factor and emission efficiency over the phase change of $\mathrm{Sb}_{2} \mathrm{~S}_{3}$ from crystalline to amorphous [150]. Another PCM, vanadium dioxide $\left(\mathrm{VO}_{2}\right)$, has been 

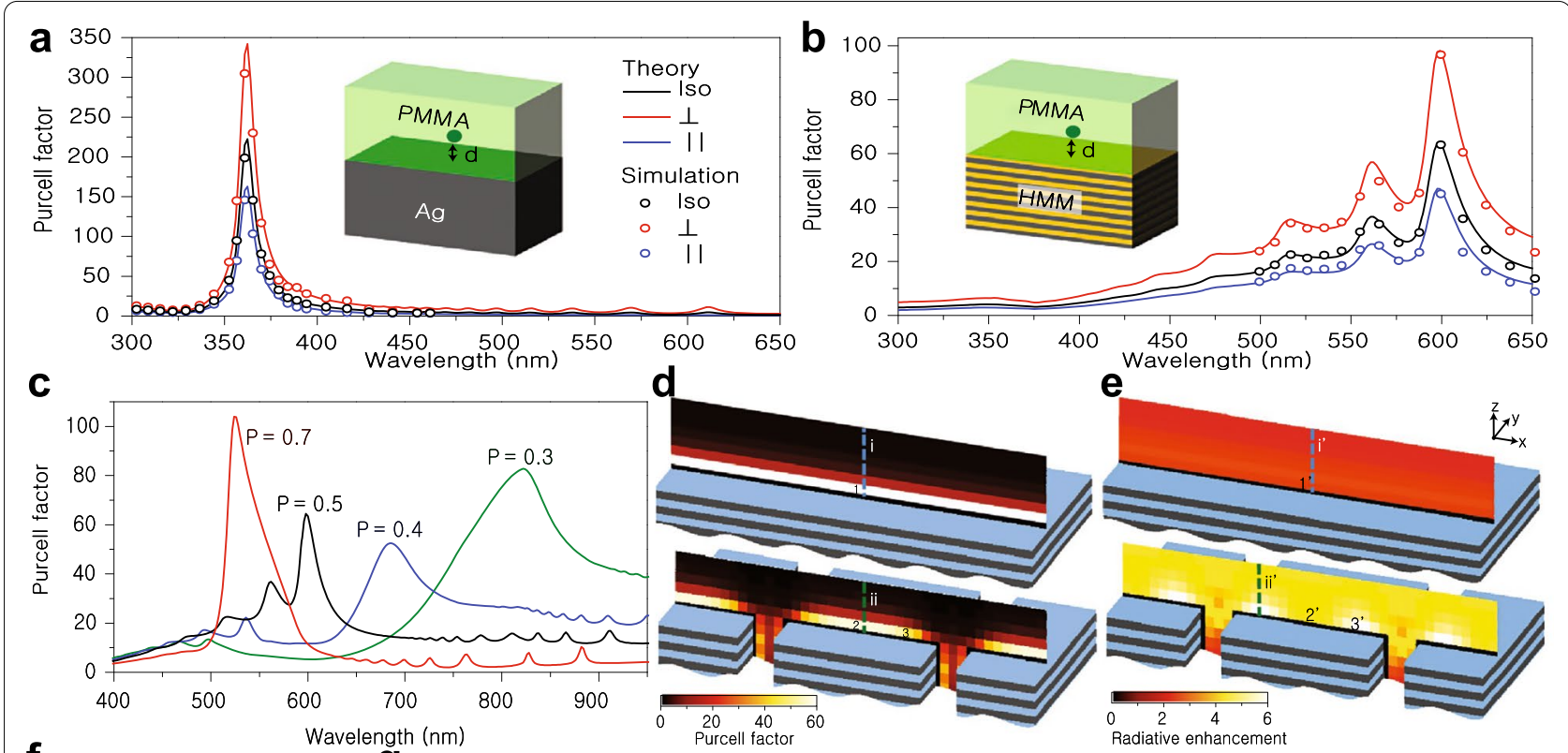

$e^{\text {e }}$

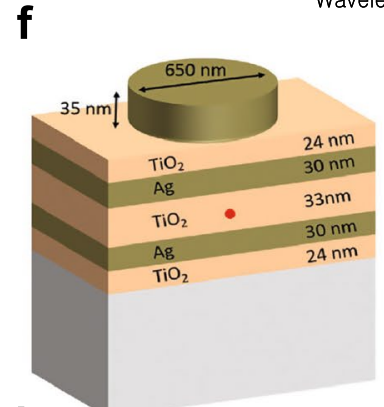

$\mathbf{h}_{22}$

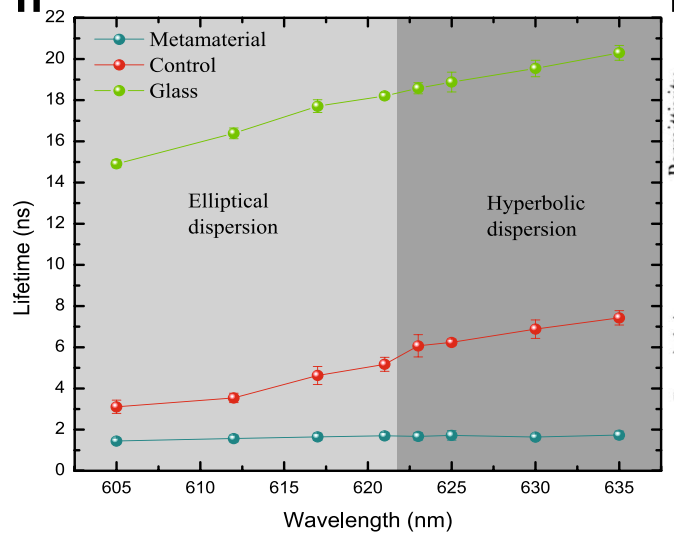

g $\begin{array}{cccc}0 & 2 & 4 & 6 \\ \text { Radiative enhancement }\end{array}$

Page 12 of 23

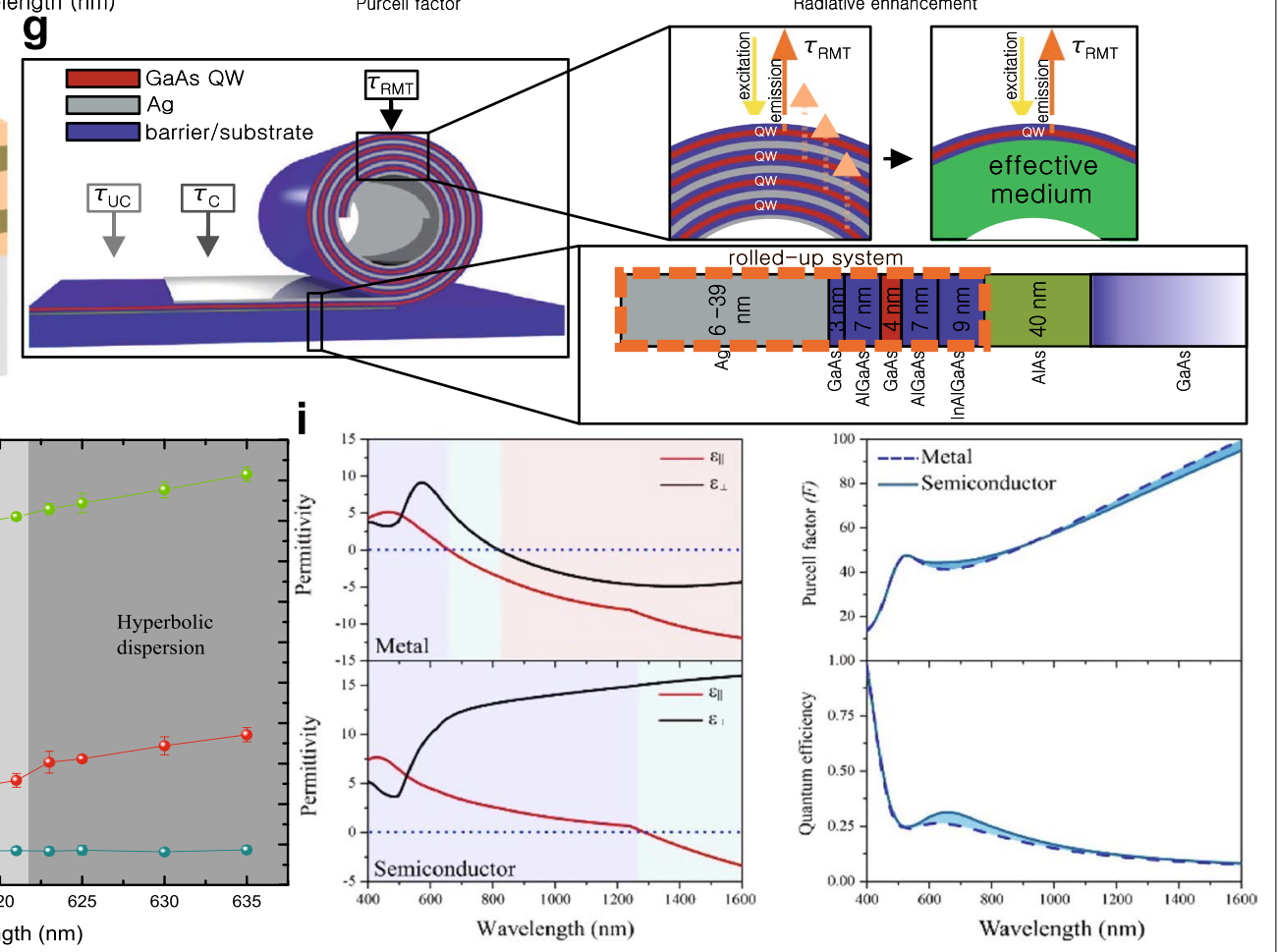

Fig. 7 Spontaneous emission engineering using HMMs. Calculated Purcell factors above $\mathbf{a}$ the Ag layer and $\mathbf{b}$ a HMM composed of Ag and silicon (Si). c Tunable characteristics of the Purcell factor according to the filling ratio of HMMs. Color map of $\mathbf{d}$ Purcell factor and $\mathbf{e}$ radiative enhancement from a multilayer HMM (top) and nanopatterned HMM (bottom). Schematic of the outcoupling of a HMM $\mathbf{f}$ with a cylindrical antenna and $\mathbf{g}$ rolled-up structure. $\mathbf{h}$ The lifetime of emitters on different substrates; only emitters on metamaterials with a hyperbolic dispersion show a decrease of lifetime. $\mathbf{i}$ Effective permittivity of vanadium dioxide $\left(\mathrm{VO}_{2}\right)$ in metal and semiconductor phases, and the Purcell factor and quantum efficiency in both phases. a-e Adapted with permission from ref. [146], copyright 2014, NPG. f Adapted with permission from ref. [147], copyright 2020, WILEY. g Adapted with permission from ref. [148], copyright 2016, APS. h Adapted with permission from ref. [149], copyright 2012, AAAS. i Adapted with permission from ref. [150], copyright 2020, OSA 
demonstrated in a tunable HMM that was used to control the LDOS at visible and NIR wavelengths (Fig. 7i).

Besides the optical applications, hyperbolic dispersion in HMMs can be utilized in thermal emission applications [35, 172-174]. At thermal equilibrium, the black body emission in the far-field follows Plank's law. However, the high DOS of HMM can achieve super-Plankian thermal emission in the near-field that can overcome the aforementioned limit [172, 175]. Although conventional photonic crystals or surface electromagnetic excitations have been reported, the narrow bandwidth has always been the bottleneck. On the other hand, non-resonant media with hyperbolic dispersion have provided thermal emission enhancement in a broad bandwidth [176-178], and various applications of emitters and radiative thermal diode have been reported [179-182]. More applications are expected using artificial HMMs and 2D materials.

\subsection{HMM-based sensors and absorbers}

HMMs have been investigated for the use as ultrasensitive optical sensors and broadband absorbers [23, 127, 183-185]. The hyperbolic dispersion of HMMs supports large wave vectors that have a large momentum and can propagate inside or on the surface of HMMs. The large wave vector components in HMMs are non-radiative, so a grating or prism can be used as a coupler [50,77]. The bulk plasmon polaritons in HMMs can be excited by the coupler, and the excited mode can stimulate multimode resonances that have high quality factors and can be utilized in ultrasensitive optical sensors [77].

Multilayer or nanorod structures of HMMs have been applied as refractive-index sensors for the detection of biomolecules. Multilayer HMMs composed of $\mathrm{Au}$ and $\mathrm{Al}_{2} \mathrm{O}_{3}$ with a 2D patterned grating have been used as a biosensor to sense wavelength and angular modulations with high sensitivity in the visible and NIR regions [186]. The measured sensitivity was $30,000 \mathrm{~nm} / \mathrm{RIU}$ (refractive index unit), which is strong enough to detect small molecules at concentrations less than picomoles. A HMM sensor based on porous nanorods with high sensitivity to refractive-index changes near the HMM medium has also been demonstrated [185]. In addition, HMM sensors have also used chiral structures and PCMs [187, 188]. A numerical investigation of a hyperbolic chiral-structure thin film as an angular-modulation sensor showed a sensitivity [187]. A tunable HMM as a reconfigurable plasmonic sensor has been proposed by structuring a hybrid HMM platform with TiN or graphene and $\mathrm{Sb}_{2} \mathrm{~S}_{3}$ as the PCM [188]. The $\mathrm{Sb}_{2} \mathrm{~S}_{3}$-TiN HMMs sensors have demonstrated significant sensitivity to detect extremely low concentrations of molecules below $1 \times 10^{-15} \mathrm{M}$.

Broadband absorbers using HMMs have also been proposed. The broadband high absorption of incident light has been achieved using HMM waveguide structures whereby the large attenuation is attributable to slowlight modes [189-191]. Typical sawtooth and pyramidal waveguide HMM structures have been extensively studied [192-195]. In numerical [193] and experimental [195] demonstrations, tapered waveguide HMM structures have demonstrated outstanding absorption of $>95 \%$ in the mid-infrared (MIR) region from 3 to $5.5 \mu \mathrm{m}$, as well as in the visible and NIR regions. Recently, non-tapered HMM broadband absorbers with a uniform height have been reported [196, 197]. A multilayer HMM made up of a Au-zinc sulfide ( $\mathrm{ZnS}$ ) grating structure has been used to demonstrate wide-angle and broadband absorption in the MIR region as a result of multiple slow-light modes. A non-tapered HMM broadband absorber in the MIR region has been demonstrated and applied to radiative cooling [197] to radiate heat through the atmosphere without consuming energy [198-201].

\subsection{Natural hyperbolic metamaterials}

The homogenized anisotropic responses of metamaterials are obtained by the engineering of artificial structures, such as dielectric and metallic multilayers [49] and periodic nanorod arrays [26]. However, these approaches have some limitations. The first is the homogenization model of metamaterials, i.e., the EMT as discussed in Sects. 2.2 and 2.3. Such simple descriptions overlook nonlocal effects induced by the intrinsic near-field coupling between two neighboring structures, especially close to resonant frequencies. Bragg scattering in inhomogeneous multilayers limit the maximum value of possible wave vectors to the boundary of its first Brillion zones (i.e., $\pi / \Lambda$, where $\Lambda$ is the periodicity of unit cells). In that sense, $\Lambda$ is required to be as small as possible, which is not practical considering the limited resolution of fabrication capabilities. Moreover, HMMs with plasmonic media have unavoidable Ohmic losses which makes them intrinsically lossy due to electron-electron scattering. To overcome these problems, recent developments in materials science have revealed many natural low-dimensional nanomaterials as alternative candidates to HMM platforms for various applications.

The first class of optical hyperbolicity consists of hyperbolic plasmon polaritons (HPPs) in 2D materials. Polaritons are quasiparticles of the collective excitation of strongly coupled light and matter [202]; plasmon polaritons are due to the coupling between the light and free or quasi-free electrons. Many natural materials can support HPPs, including black phosphorus (BP) [203-207], the semimetal tungsten ditelluride $\left(\mathrm{WTe}_{2}\right)$ and others $[208$, 209], which present reduced symmetry (orthorhombic crystal structure for $\mathrm{BP}$ and $\mathrm{WTe}_{2}$ ) in their atomic orientations. When free carriers are injected, the electron 

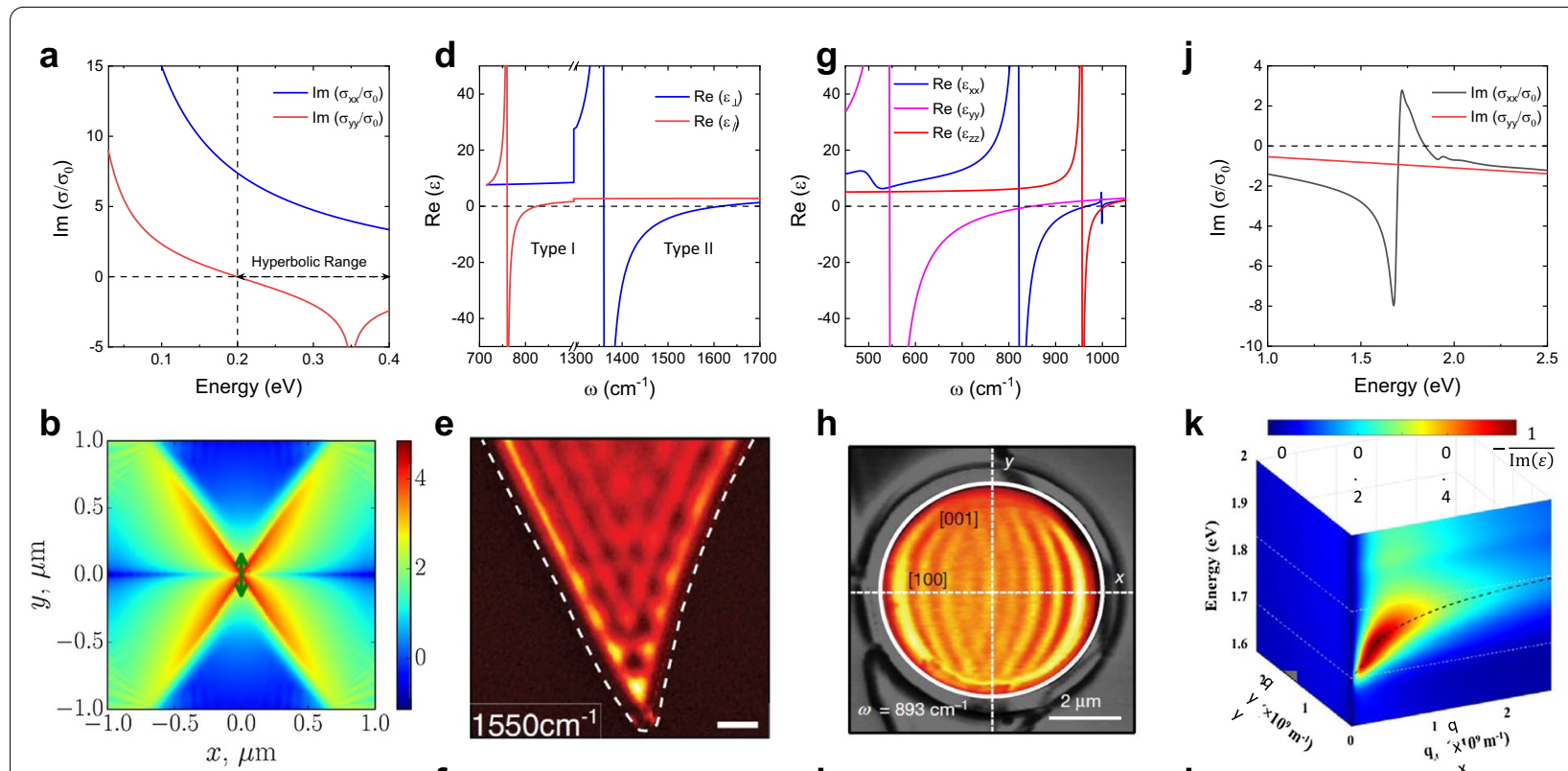

h
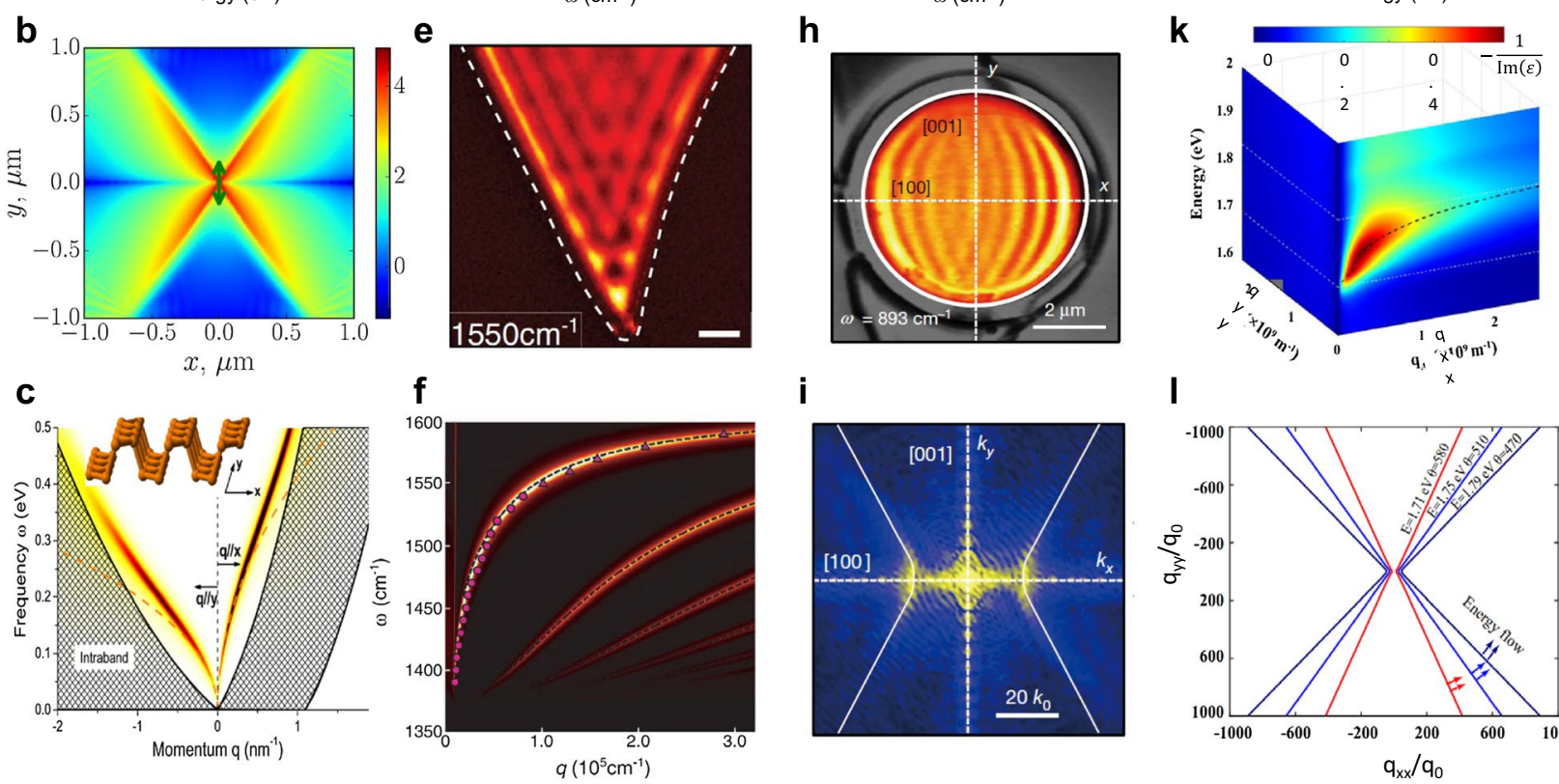

i
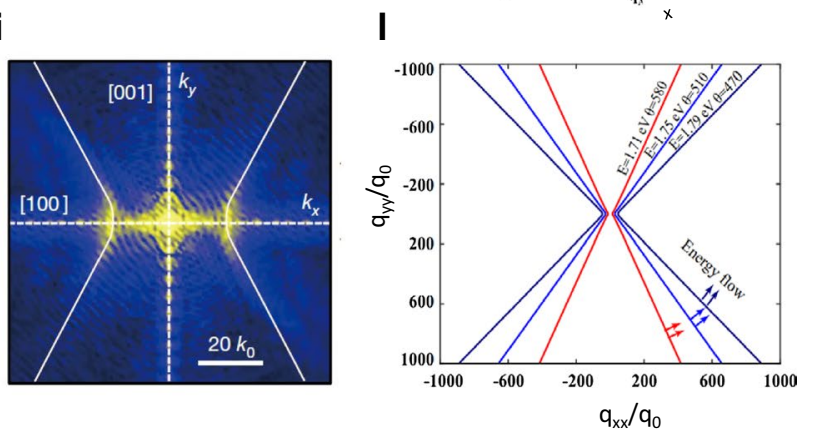

Fig. 8 Hyperbolic responses in natural materials a The surface conductivity of black phosphorus along two distinct directions. Here, $\eta_{x x}=\eta_{y y}=$ $0.01 \mathrm{eV}, m_{x x}=0.2 m_{0}, m_{y y}=m_{0}, \omega_{x x}=1 \mathrm{eV}, \omega_{y y}=0.35 \mathrm{eV}, s_{x x}=1.7 \sigma_{0}, s_{y y}=3.7 \sigma_{0} ; m_{0}$ is the free electron mass, and $\sigma_{0}=e^{2} / 4 \hbar$. The electron density is $10^{14} \mathrm{~cm}^{-2}$. b The numerically obtained distribution of HPPs at $0.3 \mathrm{eV}$ (in logscale of $|E|$ ). $\mathbf{c}$ The dispersion along $x$ and $y$ directions, for electron doping of $1013 \mathrm{~cm}^{-2}$, and $q$ is the momentum along $x$ (right axis) and $y$ (left axis) directions. $\mathbf{d}$ Permittivity of hexagonal boron nitride (hBN). e Real-space imaging of HPhPs in hBN at $1.550 \mathrm{~cm}^{-1}$ for hBN with thickness of $256 \mathrm{~nm}$. The scale bar is $800 \mathrm{~nm}$. f Dispersions of HPhPs in hBN slab with thickness $134 \mathrm{~nm}$ on top of the $\mathrm{SiO}_{2}$ substrate. Black dashed curves: theoretical values according to Eq. (9); circles and rectangles: measured data. $\mathbf{g}$ Permittivity of alpha-phase molybdenum trioxide $\left(\alpha-\mathrm{MoO}_{3}\right) \cdot \mathbf{h}$ Real-space imaging of $\mathrm{HPhPs}$ in $\boldsymbol{\alpha}-\mathrm{MoO}_{3}$ nanodisk with thickness of $144 \mathrm{~nm}$. $\mathbf{i}$ Experimentally obtained dispersion and analytical dispersions. $\mathbf{j}$ The normalized optical conductivity of BP along armchair and zigzag directions. $\mathbf{k}$ The loss function of HExPs in monolayer BP. The $x x$ and $y y$ mean the armchair and zigzag directions respectively. I The in-plane dispersions of HExPs in BP at different frequencies. b Adapted with permission from ref. [206], copyright 2016, APS. c Adapted with permission from ref. [205], copyright 2014, APS. e, f Adapted with permission from ref. [210], copyright 2014, AAAS. h, i Adapted with permission from ref. [47], copyright 2018, NPG. k, I Adapted with permission from ref. [211], copyright 2021, NPG

motions can differ along the different characteristic axes, i.e., zigzag direction ( $x$ axis in the inset of Fig. 8 c), and armchair direction ( $y$ axis in the inset of Fig. 8c), which induces electromagnetic anisotropy. BP has different band gaps in the two orthogonal directions, so the interband transitions and intraband transitions are correspondingly excited by the light, where the inductive $[\operatorname{Im}(\sigma)>0]$ and capacitive $[\operatorname{Im}(\sigma)<0]$ responses are induced (Fig. 8a), and cause the hyperbolic propagation of plasmons [205-207] in the infrared and terahertz ranges (Fig. 8b). Specifically, the surface conductivity can be written as

$$
\sigma_{\alpha \alpha}=\frac{i e^{2}}{\omega+i \eta_{\alpha}} \frac{n}{m_{\alpha}}+s_{\alpha}\left[\Theta\left(\omega-\omega_{\alpha}+\frac{i}{\pi} \ln \left(\frac{\omega-\omega_{\alpha}}{\omega+\omega_{\alpha}}\right)\right]\right.
$$

where $\alpha=x, y, \eta_{\alpha}, m_{\alpha}, \omega_{\alpha}$, and $s_{\alpha}$ are the relaxation time, effective mass of the carriers, onset frequency of interband transitions, and strength along $\alpha$ directions, respectively. $\Theta\left(\omega-\omega_{\alpha}\right)$ is a step function. The behaviors along the $x$ and $y$ directions differ (Fig. 8a-c). For some particular doping levels, an emerging hyperbolic frequency range with different signs of conductivity along the two directions occur (Fig. 8a), with supported hyperbolic 
in-plane propagation (Fig. 8b). This natural hyperbolicity at the surface is an ideal platform for HMSs. More recently, similar HPPs have been reported and observed in anisotropic Weyl semimetals of $\mathrm{WTe}_{2}$ [209], while other materials are yet to be explored.

The second class of natural optical hyperbolicity is composed of hyperbolic phonon polaritons (HPhPs) in polar van der Waals (vdW) materials [212, 213]. vdW nanomaterials are made of 2D atomic layers. Different interaction mechanisms and consequent interaction strength exist in atomically thin materials, including relatively weak $\mathrm{vdW}$ forces between the constituent atomic sheets, and strong Coulomb interactions of in-plane covalent bonds. Even for covalent bonds, the interaction strengths can also be dramatically different, as it depends on the participating atoms and their separation distances. Phonon polaritons (PhPs) are coupled oscillations between the light and ion lattice vibrations in materials that can be polarized (polar dielectrics); [214] rich anisotropic $\mathrm{PhP}$ responses can be found in layered vdW materials. The first example is uniaxial $\mathrm{hBN}$, which has a so-called out-of-plane hyperbolicity $\left[\varepsilon_{x x}=\varepsilon_{y y}=\varepsilon_{\perp}, \varepsilon_{z z}=\varepsilon_{\|}, \varepsilon_{\perp} \neq \varepsilon_{\|}, \operatorname{Re}\left(\varepsilon_{\perp}\right) \cdot \operatorname{Re}\left(\varepsilon_{\|}\right)<0\right]$ within the Reststrahlen bands. Here, the Reststrahlen bands represent the frequency range between the longitudinal and transverse optical phonons of the materials which, for hBN, contains both Type I $\left(\varepsilon_{\|}<0\right.$ and $\varepsilon_{\perp}>0$, $\left.746-819 \mathrm{~cm}^{-1}\right)$ and Type II $\left(\varepsilon_{\|}>0\right.$ and $\varepsilon_{\perp}<0,1370-1610$ $\mathrm{cm}^{-1}$ ) hyperbolicity (Fig. 8d). For a thin hBN slab, HPhPs propagate as in a waveguide mode, with the dispersion given by the constructive interference conditions of highly confined TM modes, which are written as [210]

$$
\begin{aligned}
k_{\rho} & =q+i \kappa \\
& =\frac{\tan \theta}{d}\left[\operatorname{atan}\left(-\frac{\varepsilon_{\text {sup }}}{\varepsilon_{\perp} \tan \theta}\right)+\operatorname{atan}\left(-\frac{\varepsilon_{\text {sub }}}{\varepsilon_{\perp} \tan \theta}\right)+\pi l\right],
\end{aligned}
$$

where $d$ is the thickness of the hBN and $\varepsilon_{\text {sup }}\left(\varepsilon_{\text {sub }}\right)$ are the dielectric constants of the superstrate and substrate, the integer $l$ is the mode index, $\tan \theta(\omega)=i \frac{\sqrt{\varepsilon_{1}}}{\sqrt{\varepsilon_{\|}}}$, and $k_{\rho}=\sqrt{k_{x}^{2}+k_{y}^{2}}$ is the in-plane wave vector. Using recently developed real-space nanoimaging techniques (scattering-type scanning near-field optical microscopy), one can easily map out the spatial distribution of HPhPs (Fig. 8e) and thus their dispersion features (Fig. 8f). The HPhPs in hBN capture the most of exotic properties of HMMs, such as large field confinement and directional propagation [31, 92]. Moreover, the maximum possible wave vector supported in $\mathrm{hBN}$ is limited by the size of its atomic lattice, which is several orders of magnitude larger than that of HMMs made of artificial structures. Most importantly, fundamentally distinct from plasmonic systems, HPhPs are, in principle, immune to Ohmic losses due to the absence of electron-electron scattering. Therefore, they provide a promising resource on which to study many applications of HMMs that require low-loss.

However, HPhPs in uniaxial hBN do not carry anisotropic responses at the interface because the optical axis of exfoliated $\mathrm{hBN}$ is always perpendicular to the interface. To mimic the functionalities of hyperbolic responses over a surface with $\mathrm{PhPs}$, some other materials have recently been discovered. One example is alpha-phase molybdenum trioxide $\left(\alpha-\mathrm{MoO}_{3}\right)$, a biaxial vdW material, which can be endowed with in-plane hyperbolic responses $\left[\operatorname{Re}\left(\varepsilon_{x x}\right) \cdot \operatorname{Re}\left(\varepsilon_{y y}\right)<0\right]$ within certain Reststrahlen bands (for example, around $900 \mathrm{~cm}^{-1}$ as shown in Fig. 8g) [46, $47,215,216]$. Using real-space nanoimaging techniques, one can probe the in-plane HPhPs distribution at the thin $\alpha-\mathrm{MoO}_{3}$ slab (Fig. 8h) and can experimentally retrieve the dispersion (Fig. 8i), which agrees excellently with the analytical dispersion. Here, the analytical dispersion is obtained by treating the thin slab as the surface with the conductivity tensor connected to the permittivity tensor [217]. Such approaches directly indicate that the thin in-plane anisotripic vdW films can be ideal platforms on which to study infrared HMSs, and the further incorporation of many other possible materials including $\alpha-\mathrm{V}_{2} \mathrm{O}_{5}$ [48] can add more opportunities to achieve the on-demand manipulation of light in natural hyperbolic materials.

Those HPPs and HPhPs in 2D materials mostly reside in middle-infrared or terahertz frequency ranges, as constrained by either the strength of optical excitations (for plasmon) or the intrinsic lattice vibration resonance (for phonon). For the hyperbolicity in the visible, the exciton polaritons (ExPs) should be visited. Excitons are another elementary material excitations in a form of bound electron-hole pairs by Coulomb interactions, forming the ExPs when coupled to electromagnetic waves. Such ExPs are generic and can exist in most 2D semiconductors, that hold the band gap in the visible and near-infrared, including perovskites, transition metal-dichalcogenides, BP and others. They can also present the extreme anisotropy in the presence of strong atomic lattice asymmetry. For instance, it is revealed that the 2D hybrid perovskites made of alternating layered organic and inorganic sublattices can mimic the artificially metal-dielectric HMMs and thus present the out-of-plane hyperbolic responses [218]. More interestingly, BP can also hold in-plane hyperbolic ExPs (HExPs) [211]. As discussed, BP shows the thickness-dependent band gap, spanning from the visible and near-infrared, and the in-plane distinct lattice arrangements for different principal directions. The excitons therein are hence anisotropic [219] and can be extreme for some specific cases. Figure $8 j$ plots the optical conductivity of monolayer BP in zigzag and armchair 
directions, showing the HExPs near 1s excitons. Figure $8 \mathrm{k}$ shows the loss function, defined by $-1 / \operatorname{Im}(\varepsilon(\mathbf{q}, \omega))$ while $\mathbf{q}$ and $\omega$ represent the wave vector and frequency and $\varepsilon=1+i \sigma / \omega d \varepsilon_{0}$ is the permittivity of BP with thickness $d$, of monolayer BP along two distinctive directions. One can clearly see the distinctive excitonic resonant loss in zigzag and armchair directions, as the signature of the in-plane hyperbolicity (Fig. 81). In general, HExPs can host strong light confinement for enhanced lightmatter interactions due to the open IFC and, more importantly, several other unique figures of merits. The first one is the flexible tunability, as the result of sensitive band gaps, taking BP for example, to the conditions such as layer numbers, strain and electrostatic gating. Secondly, excitons can become radiative. The photonic LDOS of those radiative excitons buried in the intrinsic hyperbolic media can be even more enhanced, compared to that of an emitter in the adjacent, promising more efficient energy harvesting and light-emitting applications [218]. Last, more advanced pathway toward the excitonic hyperbolicity can also exist in, for example, Rydberg excitons, which can be programmed by the transient photoexcitations. We note that the HExPs are only recently identified, while more future efforts on its fundamental mechanisms, materials exploration, experimental mappings, optical engineering and applications should definitely be made in the community.

To close this section, we will briefly discuss more possibilities of natural hyperbolic materials, their applications and limitations which may be possible to overcome in combination with HMMs. Firstly, beyond the routes mentioned above, other methods to achieve natural hyperbolicity exist, including photoexcitation of electron-hole pairs in layered transition-metal dichalcogenides for broadband anisotropy [220], and materials with reduced symmetry [221, 222], to name just a few. Secondly, since the hyperbolic responses in natural materials have lower loss and promise much larger momentum (meaning higher field confinement), tremendous nanotechnologies are thus possible, such as imaging [31, 92, 223], hyperlensing [67], biosensing [224, 225], waveguide [226], on-chip electro-optic modulator [227], and many others [54, 132, 228]. Thirdly, we remark that those hyperbolic responses can exist in beyond 2D materials, while the bulk media can also hold the hyperbolicity. For instance, the HPhPs are recently found in calcite, a polar crystal that has been long studied for more than three centuries. The infrared calcite polaritons feature the raylike and ultra-long propagation distance with a titled wavefront even at room-temperature [44]. The uniqueness of $2 \mathrm{D}$ materials is their atomic thinness, important for the highly compact and integrated devices, while its challenges of large-scale fabrication is formidable. The bulk media such as calcite can be easily obtained and engineered, offering the readily commercial platform for hyperbolic photonic devices, but may suffer from the large volume and poor compactness. Lastly, those hyperbolicities are intrinsically bounded by pristine forms of material candidates and hence have fixed working frequency ranges, which impose a limit on the working frequency ranges of various exciting applications. Further application of the philosophy of metamaterials, i.e., to mold electromagnetic responses by engineering sub-wavelength structures, could yield architectures of natural hyperbolic materials that induce exotic wave phenomena, such as infrared HMSs in nanostructured $\mathrm{hBN}[66,67]$, topological polaritons in twisted $\left(\alpha-\mathrm{MoO}_{3}\right)$ bilayers [217], stacked multilayers for heterostructures $[65,128,229-232]$, and others [233].

\section{Conclusion and outlook}

HMMs have extreme anisotropy and can support high$k$ waves; distinctive features that have been exploited to demonstrate various optical applications. In this review, we have focused on the fundamentals of HMMs and HMSs, and reviewed their applications. First, the fundamental background of hyperbolic dispersion in the view of bulk and 2D planar structures was discussed, and then various applications of HMMs were introduced. The wide range of applications of HMMs highlights the potential of functional optical devices and platforms.

The development of hyperbolic media that will lead to the improvement of influential optical devices is more extensive than the contents of this review. For example, photostability, low-loss organic HMMs and super-resolution imaging platform using HMMs are being actively investigated [90, 234-238]. The propagation of light in HMMs can be applied to various optical components for collimation [70, 239], splitters [240243], airy beams [244, 245], and even with absorption and scattering for sensing and cloaking [246]. Furthermore, lasers and hypercrystals that use HMMs are also being actively studied [247-250], and cavity structures capable of resonance regardless of their size and scale are being investigated [143]. HMMs have also been applied to various physical phenomena such as paritytime symmetry [251], mechanical properties [252], magneto-optical Kerr effect [253], nonlinear responses [254], chiral surface waves [255], and newly-researched substances such as perovskites [256]. Exploration of surface waves at boundaries of HMMs or surface waves with hyperbolic dispersion is also a fascinating area. HMMs support a unique type of directional surface waves with either full or partial hyperbolic dispersion called Dyakonov plasmons [57, 61, 257-260]. 
Similar surface waves with hyperbolic dispersion also exist between an isotropic and anisotropic media, even when the anisotropic media has an elliptical dispersion [261, 262]. Besides, a HMS that supports electric and magnetic surface waves simultaneously has been reported recently [263]. Finally, combination of hyperbolic dispersion with other effective optical properties opens a new path to guide surface waves topologically [264-266].

To extend the range of practical applications of HMMs and HMSs, some problems must be overcome. First, the efficiency of HMMs must be increased by minimizing intrinsic Ohmic losses caused by the commonly-used metals [267]. Maximizing the efficiency could be achieved by minimizing the proportion of metal. Active or tunable devices would also be extremely valuable. In addition to the examples introduced above, various materials are expected to be evaluated as candidates for active or switchable devices that have many potential applications. Finally, straightforward and inexpensive fabrication technologies for the realization of large-area hyperbolic media and patterns would also be beneficial [268]. Recently, various designs other than the multilayer and nanowire structures are proposed such as 3D nanorod arrays [127], core-shell nanoparticles [269, 270], hyperbolic van der Waals crystals [271], and films of carbon nanotubes [272]. In addition, metasurface studies have included many attempts to achieve the large-area processing of HMMs using nanomanufacturing methods such as nanoimprinting, colloidal lithography, aerosol jets parallel printing, and spark plasma sintering [273-276]. If successful, these methods could substantially accelerate the use and development of HMMs for practical application in photonic devices.

\section{Acknowledgements}

S.S. and M.K. acknowledge the NRF Global Ph.D. fellowships (NRF2017H1A2A1043322, NRF-2017H1A2A1043204), respectively, funded by the Ministry of Education, Republic of Korea.

\section{Authors' contributions}

$\mathrm{DL}, \mathrm{SS}, J \mathrm{R}, \mathrm{GH}$, and C-WQ mainly wrote the manuscript and produced the figures. MK, TB, HC, JK, and HK provided the advices and partially involved writing the manuscript. All authors participated in the discussion and confirmed the final manuscript. JR and C-WQ guided all aspects of the work. All authors read and approved the final manuscript.

\section{Funding}

This work was financially supported by the POSCO-POSTECH-RIST Convergence Research Center program funded by POSCO, the POSTECH-Samsung Semiconductor Research Center (IO201215-08187-01) funded by Samsung Electronics, and the National Research Foundation (NRF) grant (NRF2019R1A2C3003129) funded by the Ministry of Science and ICT, Republic of Korea. C.-W.Q. acknowledges the grant (R-261-518-004-720) from Advanced Research and Technology Innovation Center (ARTIC). G. H. acknowledges the support from A*STAR under its AME Young Individual Research Grants (YIRG, No. A2084c0172).
Availability of data and materials

Not applicable.

\section{Declarations}

\section{Competing interests}

The authors declare that they have no competing interests.

\section{Author details}

${ }^{1}$ Department of Mechanical Engineering, Pohang University of Science and Technology (POSTECH), Pohang 37673, Republic of Korea. ${ }^{2}$ Department of Biomedical Engineering, Yonsei University, Wonju 26493, Republic of Korea. ${ }^{3}$ Department of Electrical and Computer Engineering, National University of Singapore, Singapore 117583 , Singapore. ${ }^{4}$ Department of Chemical Engineering, Pohang University of Science and Technology (POSTECH), Pohang 37673, Republic of Korea. ${ }^{5}$ POSCO-POSTECH-RIST Convergence Research Center for Flat Optics and Metaphotonics, Pohang 37673, Republic of Korea.

Received: 4 June 2021 Revised: 6 October 2021 Accepted: 3 November 2021

Published online: 10 January 2022

References

1. J.B. Pendry, Negative refraction makes a perfect lens. Phys. Rev. Lett. 85(18), 3966 (2000)

2. B. Walther, C. Helgert, C. Rockstuhl, F. Setzpfandt, F. Eilenberger, E.-B. Kley, F. Lederer, A. Tünnermann, T. Pertsch, Spatial and spectral light shaping with metamaterials. Adv. Mater. 24(47), 6300-6304 (2012)

3. L. Huang, X. Chen, H. Mühlenbernd, H. Zhang, S. Chen, B. Bai, Q. Tan, G. Jin, K.-W. Cheah, C.-W. Qiu, J. Li, T. Zentgraf, S. Zhang, Three-dimensional optical holography using a plasmonic metasurface. Nat. Commun. 4, $2808(2013)$

4. X. Ni, A.V. Kildishev, V.M. Shalaev, Metasurface holograms for visible light. Nat. Commun. 4, 2807 (2013)

5. L. Huang, H. Mühlenbernd, X. Li, X. Song, B. Bai, Y. Wang, T. Zentgraf, Broadband hybrid holographic multiplexing with geometric metasurfaces. Adv. Mater. 27(41), 6444-6449 (2015)

6. G. Zheng, H. Mühlenbernd, M. Kenney, G. Li, T. Zentgraf, S. Zhang, Metasurface holograms reaching $80 \%$ efficiency. Nat. Nanotechnol. 10(4), 308 (2015)

7. Z. Li, I. Kim, L. Zhang, M.Q. Mehmood, M.S. Anwar, M. Saleem, D. Lee, K.T. Nam, S. Zhang, B. Luk'yanchuk, Y. Wang, G. Zheng, J. Rho, C.-W. Qiu, Dielectric meta-holograms enabled with dual magnetic resonances in visible light. ACS Nano 11(9), 9382-9389 (2017)

8. G. Yoon, D. Lee, K.T. Nam, J. Rho, Pragmatic metasurface hologram at visible wavelength: the balance between diffraction efficiency and fabrication compatibility. ACS Photonics 5(5), 1643-1647 (2017)

9. G. Yoon, D. Lee, K.T. Nam, J. Rho, "Crypto-display" in dual-mode metasurfaces by simultaneous control of phase and spectral responses. ACS Nano 12(7), 6421-6428 (2018)

10. H.S. Khaliq, I. Kim, J. Kim, D.K. Oh, M. Zubair, K. Riaz, M.Q. Mehmood, J. Rho, Manifesting simultaneous optical spin conservation and spin isolation in diatomic metasurfaces. Adv. Opt. Mater. 9(8), 2002002 (2021)

11. I. Kim, J. Jang, G. Kim, J. Lee, T. Badloe, J. Mun, J. Rho, Pixelated bifunctional metasurface-driven dynamic vectorial holographic color prints for photonic security platform. Nat. Commun. 12, 3614 (2021)

12. J. Kim, Y. Yang, T. Badloe, I. Kim, G. Yoon, J. Rho, Geometric and physica configurations of meta-atoms for advanced metasurface holography. InfoMat 3(7), 739-754 (2021)

13. I. Kim, H. Jeong, J. Kim, Y. Yang, D. Lee, T. Badloe, G. Kim, J. Rho, Dualband operating metaholograms with heterogeneous meta-atoms in the visible and near-infrared. Adv. Opt. Mater. 9(19), 2100609 (2021)

14. M. Khorasaninejad, W.T. Chen, R.C. Devlin, J. Oh, A.Y. Zhu, F. Capasso, Metalenses at visible wavelengths: diffraction-limited focusing and subwavelength resolution imaging. Science 352(6290), 1190-1194 (2016) 
15. M. Khorasaninejad, F. Capasso, Metalenses: versatile multifunctional photonic components. Science 358(6367), eaam8100 (2017)

16. F. Aieta, P. Genevet, M.A. Kats, N. Yu, R. Blanchard, Z. Gaburro, F. Capasso, Aberration-free ultrathin flat lenses and axicons at telecom wavelengths based on plasmonic metasurfaces. Nano Lett. 12(9), 4932-4936 (2012)

17. D. Lin, P. Fan, E. Hasman, M.L. Brongersma, Dielectric gradient metasurface optical elements. Science 345(6194), 298-302 (2014)

18. A. Arbabi, E. Arbabi, Y. Horie, S.M. Kamali, A. Faraon, Planar metasurface retroreflector. Nat. Photonics 11(7), 415 (2017)

19. I. Kim, R.J. Martins, J. Jang, T. Badloe, S. Khadir, H.-Y. Jung, H. Kim, J. Kim, P. Genevet, J. Rho, Nanophotonics for light detection and ranging technology. Nat. Nanotechnol. 16(5), 508-524 (2021)

20. T. Badloe, I. Kim, Y. Kim, J. Kim, J. Rho, Electrically tunable bifocal metalens with difraction-limited focusing and imaging at visible wavelengths. Adv. Sci. 8(21), 2102646 (2021)

21. A. Poddubny, I. lorsh, P. Belov, Y. Kivshar, Hyperbolic metamaterials. Nat. Photonics 7(12), 948-957 (2013)

22. Z. Guo, H. Jiang, H. Chen, Hyperbolic metamaterials: from dispersion manipulation to applications. J. Appl. Phys. 127(7), 071101 (2020)

23. P. Huo, S. Zhang, Y. Liang, Y. Lu, T. Xu, Hyperbolic metamaterials and metasurfaces: fundamentals and applications. Adv. Opt. Mater. 7(14), 1801616 (2019)

24. Z. Liu, H. Lee, Y. Xiong, C. Sun, X. Zhang, Far-field optical hyperlens magnifying sub-diffraction-limited objects. Science 315(5819), 1686-1686 (2007)

25. J. Rho, Z. Ye, Y. Xiong, X. Yin, Z. Liu, H. Choi, G. Bartal, X. Zhang, Spherical hyperlens for two-dimensional sub-diffractional imaging at visible frequencies. Nat. Commun. 1, 143 (2010)

26. J. Yao, Z. Liu, Y. Liu, Y. Wang, C. Sun, G. Bartal, A.M. Stacy, X. Zhang, Optical negative refraction in bulk metamaterials of nanowires. Science 321(5891), 930-930 (2008)

27. S. Bang, S. So, J. Rho, Realization of broadband negative refraction in visible range using vertically stacked hyperbolic metamaterials. Sci. Rep. 9. 14093 (2019)

28. M.A. Noginov, H. Li, Y.A. Barnakov, D. Dryden, G. Nataraj, G. Zhu, C.E. Bonner, M. Mayy, Z. Jacob, E.E. Narimanov, Controlling spontaneous emission with metamaterials. Opt. Lett. 35(11), 1863-1865 (2010)

29. Z. Jacob, J.-Y. Kim, G.V. Naik, A. Boltasseva, E.E. Narimanov, V.M. Shalaev, Engineering photonic density of states using metamaterials. Appl. Phys. B 100(1), 215-218 (2010)

30. J. Sun, J. Zhou, B. Li, F. Kang, Indefinite permittivity and negative refraction in natural material: graphite. Appl. Phys. Lett. 98(10), 101901 (2011)

31. J.D. Caldwell, A.V. Kretinin, Y. Chen, V. Giannini, M.M. Fogler, Y. Francescato, C.T. Ellis, J.G. Tischler, C.R. Woods, A.J. Giles et al., Sub-diffractional volume-confined polaritons in the natural hyperbolic material hexagonal boron nitride. Nat. Commun. 5, 5221 (2014)

32. K.D. Belashchenko, M. van Schilfgaarde, V.P. Antropov, Coexistence of covalent and metallic bonding in the boron intercalation superconductor $\mathrm{MgB}_{2}$. Phys. Rev. B 64(9), 092503 (2001)

33. J. Sun, N.M. Litchinitser, J. Zhou, Indefinite by nature: from ultraviolet to terahertz. ACS Photonics 1(4), 293-303 (2014)

34. P. Shekhar, J. Atkinson, Z. Jacob, Hyperbolic metamaterials: fundamentals and applications. Nano Convergence 1(1), 14 (2014)

35. L. Ferrari, C. Wu, D. Lepage, X. Zhang, Z. Liu, Hyperbolic metamaterials and their applications. Prog. Quantum Electron. 40, 1-40 (2015)

36. L.V. Alekseyev, V.A. Podolskiy, E.E. Narimanov, Homogeneous Hyperbolic Systems for Terahertz and Far-Infrared Frequencies (Adv, Optoelectron, 2012)

37. K. Korzeb, M. Gajc, D.A. Pawlak, Compendium of natural hyperbolic materials. Opt. Express 23(20), 25406-25424 (2015)

38. D.R. Smith, J.B. Pendry, M.C. Wiltshire, Metamaterials and negative refractive index. Science 305(5685), 788-792 (2004)

39. N.I. Zheludev, The road ahead for metamaterials. Science 328(5978), 582-583 (2010)

40. Y. Liu, X. Zhang, Metamaterials: a new frontier of science and technology. Chem. Soc. Rev. 40(5), 2494-2507 (2011)

41. S.S. Kruk, Z.J. Wong, E. Pshenay-Severin, K. O'brien, D.N. Neshev, Y.S Kivshar, X. Zhang, Magnetic hyperbolic optical metamaterials. Nat. Commun. 7, 11329 (2016)
42. M. Mirmoosa, S.Y. Kosulnikov, C. Simovski, Magnetic hyperbolic metamaterial of high-index nanowires. Phys. Rev. B 94(7), 075138 (2016)

43. Y. Yang, P. Qin, B. Zheng, L. Shen, H. Wang, Z. Wang, E. Li, R. Singh, H. Chen, Magnetic hyperbolic metasurface: concept, design, and applications. Adv. Sci. 5(12), 1801495 (2018)

44. W. Ma, G. Hu, D. Hu, R. Chen, T. Sun, X. Zhang, Q. Dai, Y. Zeng, A. Alù, C.-W. Qiu et al., Ghost hyperbolic surface polaritons in bulk anisotropic crystals. Nature 596(7872), 362-366 (2021)

45. M. Esslinger, R. Vogelgesang, N. Talebi, W. Khunsin, P. Gehring, S. De Zuani, B. Gompf, K. Kern, Tetradymites as natural hyperbolic materials for the near-infrared to visible. ACS Photonics 1(12), 1285-1289 (2014)

46. Z. Zheng, N. Xu, S.L. Oscurato, M. Tamagnone, F. Sun, Y. Jiang, Y. Ke, J. Chen, W. Huang, W.L. Wilson et al., A mid-infrared biaxial hyperbolic van der Waals crystal. Sci. Adv. 5(5), eaav8690 (2019)

47. W. Ma, P. Alonso-González, S. Li, A.Y. Nikitin, J. Yuan, J. Martín-Sánchez, J. Taboada-Gutiérrez, I. Amenabar, P. Li, S. Vélez et al., In-plane anisotropic and ultra-low-loss polaritons in a natural van der Waals crystal. Nature 562(7728), 557-562 (2018)

48. J. Taboada-Gutiérrez, G. Álvarez-Pérez, J. Duan, W. Ma, K. Crowley, I. Prieto, A. Bylinkin, M. Autore, H. Volkova, K. Kimura et al., Broad spectral tuning of ultra-low-loss polaritons in a van der Waals crystal by intercalation. Nat. Mater. 19(9), 964-968 (2020)

49. B. Wood, J. Pendry, D. Tsai, Directed subwavelength imaging using a layered metal-dielectric system. Phys. Rev. B 74(11), 115116 (2006)

50. I. Avrutsky, I. Salakhutdinov, J. Elser, V. Podolskiy, Highly confined optical modes in nanoscale metal-dielectric multilayers. Phys. Rev. B 75(24), 241402 (2007)

51. M.G. Silveirinha, Nonlocal homogenization model for a periodic array of $\epsilon$-negative rods. Phys. Rev. E 73(4), 046612 (2006)

52. T.C. Choy, Effective Medium Theory: Principles and Applications, vol. 165 (Oxford University Press, Oxford, 2015)

53. J. Sun, T. Xu, N.M. Litchinitser, Experimental demonstration of demagnifying hyperlens. Nano Lett. 16(12), 7905-7909 (2016)

54. M. Kim, D. Lee, T.H. Kim, Y. Yang, H.J. Park, J. Rho, Observation of enhanced optical spin Hall effect in a vertical hyperbolic metamaterial. ACS Photonics 6(10), 2530-2536 (2019)

55. P. Evans, W. Hendren, R. Atkinson, G. Wurtz, W. Dickson, A. Zayats, R. Pollard, Growth and properties of gold and nickel nanorods in thin film alumina. Nanotechnology 17(23), 5746 (2006)

56. N. Yu, P. Genevet, M.A. Kats, F. Aieta, J.-P. Tetienne, F. Capasso, Z. Gaburro, Light propagation with phase discontinuities: generalized laws of reflection and refraction. Science 334(6054), 333-337 (2011)

57. A.V. Kildishev, A. Boltasseva, V.M. Shalaev, Planar photonics with metasurfaces. Science 339(6125), 1232009 (2013)

58. J. Gomez-Diaz, M. Tymchenko, A. Alù, Hyperbolic metasurfaces: surface plasmons, light-matter interactions, and physical implementation using graphene strips. Opt. Mater. Express 5(10), 2313-2329 (2015)

59. Y. Yermakov, D.V. Permyakov, F.V. Porubaev, P.A. Dmitriev, A.K. Samusev, I.V. Iorsh, R. Malureanu, A.V. Lavrinenko, A.A. Bogdanov, Effective surface conductivity of optical hyperbolic metasurfaces: from far-field characterization to surface wave analysis. Sci. Rep. 8, 14135 (2018)

60. J. Gomez-Diaz, A. Alu, Flatland optics with hyperbolic metasurfaces. ACS Photonics 3(12), 2211-2224 (2016)

61. A.A. High, R.C. Devlin, A. Dibos, M. Polking, D.S. Wild, J. Perczel, N.P. De Leon, M.D. Lukin, H. Park, Visible-frequency hyperbolic metasurface. Nature 522(7555), 192-196 (2015)

62. O. Yermakov, A. Ovcharenko, M. Song, A. Bogdanov, I. Iorsh, Y.S. Kivshar, Hybrid waves localized at hyperbolic metasurfaces. Phys. Rev. B 91(23), 235423 (2015)

63. J.S. Gomez-Diaz, M. Tymchenko, A. Alu, Hyperbolic plasmons and topological transitions over uniaxial metasurfaces. Phys. Rev. Lett. 114(23), 233901 (2015)

64. D. Correas-Serrano, J. Gomez-Diaz, M. Tymchenko, A. Alù, Nonlocal response of hyperbolic metasurfaces. Opt. Express 23(23), 29434-29448 (2015)

65. G. Hu, A. Krasnok, Y. Mazor, C.-W. Qiu, A. Alù, Moiré hyperbolic metasurfaces. Nano Lett. 20(5), 3217-3224 (2020)

66. P. Li, I. Dolado, F.J. Alfaro-Mozaz, F. Casanova, L.E. Hueso, S. Liu, J.H. Edgar, A.Y. Nikitin, S. Vélez, R. Hillenbrand, Infrared hyperbolic metasurface 
based on nanostructured van der Waals materials. Science 359(6378), 892-896 (2018)

67. P. Li, G. Hu, I. Dolado, M. Tymchenko, C.-W. Qiu, F.J. Alfaro-Mozaz, F. Casanova, L.E. Hueso, S. Liu, J.H. Edgar et al., Collective near-field coupling and nonlocal phenomena in infrared-phononic metasurfaces for nanolight canalization. Nat. Commun. 11, 3663 (2020)

68. J. Pendry, L. Martin-Moreno, F. Garcia-Vidal, Mimicking surface plasmons with structured surfaces. Science 305(5685), 847-848 (2004)

69. Z. Gao, L. Wu, F. Gao, Y. Luo, B. Zhang, Spoof plasmonics: from metamaterial concept to topological description. Adv. Mater. 30(31), 1706683 (2018)

70. Y. Yang, L. Jing, L. Shen, Z. Wang, B. Zheng, H. Wang, E. Li, N.-H. Shen, T. Koschny, C.M. Soukoulis et al., Hyperbolic spoof plasmonic metasurfaces. NPG Asia Mater. 9(8), e428-e428 (2017)

71. Y. Meng, H. Ma, J. Wang, M. Feng, Y. Shen, S. Qu, Hyperbolic Metasurface at Microwave Frequency for Spoof Surface Plasmon Polaritons. in 2018 International Conference on Microwave and Millimeter Wave Technology (ICMMT), (IEEE, 2018) p. 1-3.

72. S. Han, Y. Xiong, D. Genov, Z. Liu, G. Bartal, X. Zhang, Ray optics at a deep-subwavelength scale: a transformation optics approach. Nano Lett. 8(12), 4243-4247 (2008)

73. D. Lee, Y.D. Kim, M. Kim, S. So, H.-J. Choi, J. Mun, D.M. Nguyen, T. Badloe, J.G. Ok, K. Kim et al., Realization of wafer-scale hyperlens device for sub-diffractional biomolecular imaging. ACS Photonics 5(7), 2549-2554 (2017)

74. E. Abbe, Beiträge zur Theorie des Mikroskops und der mikroskopischen Wahrnehmung. Arch. Mikrosk. Anat. 9(1), 413-468 (1873)

75. M. Kim, J. Rho, Metamaterials and imaging. Nano Convergence 2(1), 22 (2015)

76. S. So, M. Kim, D. Lee, D.M. Nguyen, J. Rho, Overcoming diffraction limit: from microscopy to nanoscopy. Appl. Spectrosc. Rev. 53(2-4), 290-312 (2018)

77. K.V. Sreekanth, A. De Luca, G. Strangi, Experimental demonstration of surface and bulk plasmon polaritons in hypergratings. Sci. Rep. 3, 3291 (2013)

78. W. Cai, D.A. Genov, V.M. Shalaev, Superlens based on metal-dielectric composites. Phys. Rev. B 72(19), 193101 (2005)

79. W. Lu, S. Sridhar, Superlens imaging theory for anisotropic nanostructured metamaterials with broadband all-angle negative refraction. Phys. Rev. B 77(23), 233101 (2008)

80. Z. Jacob, L.V. Alekseyev, E. Narimanov, Optical hyperlens: far-field imaging beyond the diffraction limit. Opt. Express 14(18), 8247-8256 (2006)

81. M. Byun, D. Lee, M. Kim, Y. Kim, K. Kim, J.G. Ok, J. Rho, H. Lee, Demonstration of nanoimprinted hyperlens array for high-throughput sub-diffraction imaging. Sci. Rep. 7, 46314 (2017)

82. B.H. Cheng, Y.Z. Ho, Y.-C. Lan, D.P. Tsai, Optical hybrid-superlens hyperlens for superresolution imaging. IEEE J. Sel. Top. Quantum Electron. 19(3), 4601305-4601305 (2012)

83. S. So, J. Rho, Geometrically flat hyperlens designed by transformation optics. J. Phys. D: Appl. Phys. 52(19), 194003 (2019)

84. F. Sun, J. Fu, J. Sun, Y. Liu, Y. Jin, S. He, Planar hyper-lens with uniform predesigned magnification factor by homogeneous medium. Appl. Phys. Express 14(2), 022007022007 (2021)

85. J. Sun, M.I. Shalaev, N.M. Litchinitser, Experimental demonstration of a non-resonant hyperlens in the visible spectral range. Nat. Commun. $\mathbf{6}$ 7201 (2015)

86. A.V. Kildishev, E.E. Narimanov, Impedance-matched hyperlens. Opt. Lett. 32(23), 3432-3434 (2007)

87. G.V. Naik, V.M. Shalaev, A. Boltasseva, Alternative plasmonic materials: beyond gold and silver. Adv. Mater. 25(24), 3264-3294 (2013)

88. E. Narimanov, Hyperstructured illumination. ACS Photonics $3(6)$, 1090-1094 (2016)

89. Q. Ma, H. Qian, S. Montoya, W. Bao, L. Ferrari, H. Hu, E. Khan, Y. Wang, E.E. Fullerton, E.E. Narimanov et al., Experimental demonstration of hyperbolic metamaterial assisted illumination nanoscopy. ACS nano 12(11), 11316-11322 (2018)

90. Y.U. Lee, J. Zhao, Q. Ma, L.K. Khorashad, C. Posner, G. Li, G.B.M. Wisna, Z. Burns, J. Zhang, Z. Liu, Metamaterial assisted illumination nanoscopy via random super-resolution speckles. Nat. Commun. 12, 1559 (2021)

91. E. Yoxall, M. Schnell, A.Y. Nikitin, O. Txoperena, A. Woessner, M.B. Lundeberg, F. Casanova, L.E. Hueso, F.H. Koppens, R. Hillenbrand, Direct observation of ultraslow hyperbolic polariton propagation with negative phase velocity. Nat. Photonics 9(10), 674-678 (2015)

92. P. Li, M. Lewin, A.V. Kretinin, J.D. Caldwell, K.S. Novoselov, T. Taniguchi, K. Watanabe, F. Gaussmann, T. Taubner, Hyperbolic phonon-polaritons in boron nitride for near-field optical imaging and focusing. Nat. Commun. 6, 7507 (2015)

93. J. Duan, G. Álvarez-Pérez, A. Tresquerres-Mata, J. Taboada-Gutiérrez, K. Voronin, A. Bylinkin, B. Chang, S. Xiao, S. Liu, J. Edgar et al., Planar refraction and lensing of highly confined polaritons in anisotropic media. Nat. Commun. 12, 4325 (2021)

94. A. Nikitin, P. Alonso-González, S. Vélez, S. Mastel, A. Centeno, A. Pesquera, A. Zurutuza, F. Casanova, L. Hueso, F. Koppens et al., Real-space mapping of tailored sheet and edge plasmons in graphene nanoresonators. Nat. Photonics 10(4), 239-243 (2016)

95. N. Paul, J. Gomez-Diaz, Broadband and unidirectional plasmonic hyperlensing in drift-biased graphene. Appl. Phys. Lett. 118(9), 091107 (2021)

96. M.N. Gjerding, R. Petersen, T.G. Pedersen, N.A. Mortensen, K.S. Thygesen, Layered van der Waals crystals with hyperbolic light dispersion. Nat. Commun. 8, 320 (2017)

97. M. He, G. Iyer, S. Aarav, S. Sunku, A. Giles, T. Folland, N. Sharac, X. Sun, J. Matson, S. Liu, J. Edgar, J. Fleishcer, D. Basov, J. Caldwell, Utrahigh-resolution, label-free hyperlens imaging in the Mid-IR. Nano Lett. 21(19), 7921-7928 (2021)

98. Y. Xiong, Z. Liu, X. Zhang, A simple design of flat hyperlens for lithography and imaging with half-pitch resolution down to $20 \mathrm{~nm}$. Appl. Phys. Lett. 94(20), 203108 (2009)

99. G. Liang, C. Wang, Z. Zhao, Y. Wang, N. Yao, P. Gao, Y. Luo, G. Gao, Q. Zhao, $X$. Luo, Squeezing bulk plasmon polaritons through hyperbolic metamaterials for large area deep subwavelength interference lithography. Adv. Opt. Mater. 3(9), 1248-1256 (2015)

100. M. Kim, S. So, K. Yao, Y. Liu, J. Rho, Deep sub-wavelength nanofocusing of UV-visible light by hyperbolic metamaterials. Sci. Rep. 6, 38645 (2016)

101. T. Xu, Y. Zhao, J. Ma, C. Wang, J. Cui, C. Du, X. Luo, Sub-diffraction-limited interference photolithography with metamaterials. Opt. Express 16(18), 13579-13584 (2008)

102. J. Sun, N.M. Litchinitser, Toward practical, subwavelength, visible-light photolithography with hyperlens. ACS Nano 12(1), 542-548 (2018)

103. S. Ishii, A.V. Kildishev, E. Narimanov, V.M. Shalaev, V.P. Drachev, Subwavelength interference pattern from volume plasmon polaritons in a hyperbolic medium. Laser Photonics Rev. 7(2), 265-271 (2013)

104. J.-H. Seo, J.H. Park, S.-I. Kim, B.J. Park, Z. Ma, J. Choi, B.-K. Ju, Nanopatterning by laser interference lithography: applications to optical devices. J. Nanosci. Nanotechnol. 14(2), 1521-1532 (2014)

105. H.H. Solak, Nanolithography with coherent extreme ultraviolet light. J. Phys. D: Appl. Phys. 39(10), R171 (2006)

106. Y.-C. Cheng, A. Isoyan, J. Wallace, M. Khan, F. Cerrina, Extreme ultraviolet holographic lithography: initial results. Appl. Phys. Lett. 90(2), 023116 (2007)

107. P. Zhu, H. Shi, L.J. Guo, SPPs coupling induced interference in metal/ dielectric multilayer waveguides and its application for plasmonic lithography. Opt. Express 20(11), 12521-12529 (2012)

108. X. Chen, C. Zhang, F. Yang, G. Liang, Q. Li, L.J. Guo, Plasmonic lithography utilizing epsilon near zero hyperbolic metamaterial. ACS Nano 11(10), 9863-9868 (2017)

109. Y. Liu, X. Zhang, Metasurfaces for manipulating surface plasmons. Appl. Phys. Lett. 103(14), 141101 (2013)

110. G.V. Naik, J. Liu, A.V. Kildishev, V.M. Shalaev, A. Boltasseva, Demonstration of Al:ZnO as a plasmonic component for near-infrared metamaterials. Proc. Natl. Acad. Sci. USA. 109(23), 8834-8838 (2012)

111. A.J. Hoffman, L. Alekseyev, S.S. Howard, K.J. Franz, D. Wasserman, V.A. Podolskiy, E.E. Narimanov, D.L. Sivco, C. Gmachl, Negative refraction in semiconductor metamaterials. Nat. Mater. 6(12), 946-950 (2007)

112. R.A. Shelby, D. Smith, S. Nemat-Nasser, S. Schultz, Microwave transmission through a two-dimensional, isotropic, left-handed metamaterial. Appl. Phys. Lett. 78(4), 489-491 (2001)

113. J. Valentine, S. Zhang, T. Zentgraf, E. Ulin-Avila, D.A. Genov, G. Bartal, X Zhang, Three-dimensional optical metamaterial with a negative refractive index. Nature 455(7211), 376-379 (2008)

114. S. Zhang, Y.-S. Park, J. Li, X. Lu, W. Zhang, X. Zhang, Negative refractive index in chiral metamaterials. Phys. Rev. Lett. 102(2), 023901 (2009) 
115. J. Pendry, A chiral route to negative refraction. Science 306(5700), 1353-1355 (2004)

116. B. Kanté, Y.-S. Park, K. O'Brien, D. Shuldman, N.D. Lanzillotti-Kimura, Z.J. Wong, X. Yin, X. Zhang, Symmetry breaking and optical negative index of closed nanorings. Nat. Commun. 3, 1180 (2012)

117. G. Viktor, The electrodynamics of substances with simultaneously negative values of $\varepsilon$ and $\mu$. Sov. Phys. Usp. 10(4), 509 (1968)

118. R.A. Shelby, D.R. Smith, S. Schultz, Experimental verification of a negative index of refraction. Science 292 (5514), 77-79 (2001)

119. A.A. Houck, J.B. Brock, I.L. Chuang, Experimental observations of a lefthanded material that obeys Snell's law. Phys. Rev. Lett. 90(13), 137401 (2003)

120. J. Jiang, X. Lin, B. Zhang et al., Broadband negative refraction of highly squeezed hyperbolic polaritons in 2D materials. Research $\mathbf{2 0 1 8}$ 2532819 (2018)

121. Q. Chen, Y. Yang, L. Zhang, J. Chen, M. Li, X. Lin, R. Li, Z. Wang, B. Zhang, $H$. Chen, Negative refraction of ultra-squeezed in-plane hyperbolic designer polaritons. Photonics Research 9(8), 1540-1549 (2021)

122. H.N. Krishnamoorthy, B. Gholipour, N.I. Zheludev, C. Soci, A non-volatile chalcogenide switchable hyperbolic metamaterial. Adv. Opt. Mater. 6(19), 1800332 (2018)

123. G. Pawlik, K. Tarnowski, W. Walasik, A.C. Mitus, I. Khoo, Liquid crystal hyperbolic metamaterial for wide-angle negative-positive refraction and reflection. Opt. Lett. 39(7), 1744-1747 (2014)

124. C. Cortes, W. Newman, S. Molesky, Z. Jacob, Quantum nanophotonics using hyperbolic metamaterials. J. Opt. 14(6), 063001 (2012)

125. H. Cho, S. So, T. Badloe, S. Bang, J. Rho, Critical layer thickness analysis of vertically stacked hyperbolic metamaterials for effective negative refraction generation. Adv. Theory Simul. 3(11), 2000138 (2020)

126. H. Cho, Y. Yang, D. Lee, S. So, J. Rho, Experimental demonstration of broadband negative refraction at visible frequencies by critical layer thickness analysis in a vertical hyperbolic metamaterial. Nanophotonics 10(15), 3871-3877 (2021)

127. C. Li, J. Liu, Z. Wu, Z. Chen, J. Jiang, Z. Dou, X. Liu, M. Fu, D. He, Y. Wang, Hyperbolic metamaterials fabricated using 3D assembled nanorod arrays and enhanced photocatalytic performance. Adv. Opt. Mater. (in press).

128. X. Lin, Y. Yang, N. Rivera, J.J. López, Y. Shen, I. Kaminer, H. Chen, B. Zhang, J.D. Joannopoulos, M. Soljačić, All-angle negative refraction of highly squeezed plasmon and phonon polaritons in graphene-boron nitride heterostructures. Proc. Natl. Acad. Sci. USA 114(26), 6717-6721 (2017)

129. K. Sreekanth, A. De Luca, G. Strangi, Negative refraction in graphenebased hyperbolic metamaterials. Appl. Phys. Lett. 103(2), 023107 (2013)

130. H. Gao, Z. Wang, X. Ma, X. Zhang, W. Li, M. Zhao, Hyperbolic dispersion and negative refraction in a metal-organic framework $\mathrm{Cu}-\mathrm{BHT}$. Phys. Rev. Mater. 3(6), 065206 (2019)

131. O. Takayama, J. Sukham, R. Malureanu, A.V. Lavrinenko, G. Puentes, Photonic spin Hall effect in hyperbolic metamaterials at visible wavelengths. Opt. Lett. 43(19), 4602-4605 (2018)

132. M. Kim, D. Lee, H. Cho, B. Min, J. Rho, Spin Hall effect of light with nearunity efficiency in the microwave. Laser Photonics Rev. 15(2), 2000393 (2021)

133. M. Onoda, S. Murakami, N. Nagaosa, Hall effect of light. Phys. Rev. Lett. 93(8), 083901 (2004)

134. K.Y. Bliokh, Y.P. Bliokh, Conservation of angular momentum, transverse shift, and spin Hall effect in reflection and refraction of an electromagnetic wave packet. Phys. Rev. Lett. 96(7), 073903 (2006)

135. O. Hosten, P. Kwiat, Observation of the spin Hall effect of light via weak measurements. Science 319(5864), 787-790 (2008)

136. M. Kim, D. Lee, T.H.-Y. Nguyen, H.-J. Lee, G. Byun, J. Rho, Total reflectioninduced efficiency enhancement of the spin Hall effect of light. ACS Photonics 8(9), 2705-2712 (2021)

137. M. Kim, D. Lee, J. Rho, Spin Hall effect under arbitrarily polarized or unpolarized light. Laser Photonics Rev. 15(7), 2100138 (2021)

138. X. Yin, Z. Ye, J. Rho, Y. Wang, X. Zhang, Photonic spin Hall effect at metasurfaces. Science 339(6126), 1405-1407 (2013)

139. M. Kim, D. Lee, B. Ko, J. Rho, Diffraction-induced enhancement of optical spin Hall effect in a dielectric grating. APL Photonics 5(6), 066106 (2020)

140. T. Tang, Y. Zhang, J. Li, L. Luo, Spin hall effect enhancement of transmitted light through an anisotropic metamaterial slab. IEEE Photonics J. 9(4), 1-10 (2017)
141. T. Tang, C. Li, L. Luo, Enhanced spin Hall effect of tunneling light in hyperbolic metamaterial waveguide. Sci. Rep. 6, 30762 (2016)

142. H. Chen, S. Zhou, G. Rui, Q. Zhan, Magnified photonic spin-Hall effect with curved hyperbolic metamaterials. J. Appl. Phys. 124(23), 233104 (2018)

143. S.C. Indukuri, J. Bar-David, N. Mazurski, U. Levy, Ultrasmall mode volume hyperbolic nanocavities for enhanced light-matter interaction at the nanoscale. ACS Nano 13(10), 11770-11780 (2019)

144. E. Fermi, Quantum theory of radiation. Rev. Mod. Phys. 4. 87-132 (1932)

145. D. J. Roth, A. V. Zayats, Spontaneous emission inside hyperbolic metamaterials. Ph.D. thesis, King's College London (2017)

146. D. Lu, J.J. Kan, E.E. Fullerton, Z. Liu, Enhancing spontaneous emission rates of molecules using nanopatterned multilayer hyperbolic metamaterials. Nat. Nanotechnol. 9(1), 48-53 (2014)

147. A. Kala, F.A. Inam, S.-A. Biehs, P.Vaity, V.G. Achanta, Hyperbolic metamaterial with quantum dots for enhanced emission and collection efficiencies. Adv. Opt. Mater. 8(15), 2000368 (2020)

148. K.M. Schulz, H. Vu, S. Schwaiger, A. Rottler, T. Korn, D. Sonnenberg, T. Kipp, S. Mendach, Controlling the spontaneous emission rate of quantum wells in rolled-up hyperbolic metamaterials. Phys. Rev. Lett. 117 085503 (2016)

149. H.N.S. Krishnamoorthy, Z. Jacob, E. Narimanov, I. Kretzschmar, V.M. Menon, Topological transitions in metamaterials. Science 336(6078), 205-209 (2012)

150. S.K. Chamoli, M. ElKabbash, J. Zhang, C. Guo, Dynamic control of spontaneous emission rate using tunable hyperbolic metamaterials. Opt. Lett. 45(7), 1671-1674 (2020)

151. E.K. Tanyi, N. Hong, T. Sawyer, J.D.B.V. Schenck, G. Giesbers, O. Ostroverkhova, L.-J. Cheng, Strong exciton-plasmon coupling in dye-doped film on a planar hyperbolic metamaterial. Opt. Lett. 45(24), 6736-6739 (2020)

152. Y.D. Jang, J.S. Baek, V. Devaraj, M.D. Kim, J.D. Song, Y. Wang, X. Zhang, D. Lee, Deterministic coupling of epitaxial semiconductor quantum dots to hyperbolic metamaterial. Optica 5(7), 832-835 (2018)

153. K.J. Lee, Y.U. Lee, S.J. Kim, P. André, Hyperbolic dispersion dominant regime identified through spontaneous emission variations near metamaterial interfaces. Adv. Mater. Interfaces 5(8), 1701629 (2018)

154. J. Kim, V.P. Drachev, Z. Jacob, G.V. Naik, A. Boltasseva, E.E. Narimanov, V.M. Shalaev, Improving the radiative decay rate for dye molecules with hyperbolic metamaterials. Opt. Express 20(7), 8100-8116 (2012)

155. M.Y. Shalaginov, S. Ishii, J. Liu, J. Liu, J. Irudayaraj, A. Lagutchev, A.V. Kildishev, V.M. Shalaev, Broadband enhancement of spontaneous emission from nitrogen-vacancy centers in nanodiamonds by hyperbolic metamaterials. Appl. Phys. Lett. 102(17), 173114 (2013)

156. D. Lee, M. Kim, J. Lee, B. Ko, H.J. Park, J. Rho, Angular selection of transmitted light and enhanced spontaneous emission in grating-coupled hyperbolic metamaterials. Opt. Express 29(14), 21458-21472 (2021)

157. W.D. Newman, C.L. Cortes, Z. Jacob, Enhanced and directional singlephoton emission in hyperbolic metamaterials. J. Opt. Soc. Am. B 30(4) 766-775 (2013)

158. T. Tumkur, G. Zhu, P. Black, Y.A. Barnakov, C.E. Bonner, M.A. Noginov, Control of spontaneous emission in a volume of functionalized hyperbolic metamaterial. Appl. Phys. Lett. 99(15), 151115 (2011)

159. T. Galfsky, H.N.S. Krishnamoorthy, W. Newman, E.E. Narimanov, Z. Jacob, V.M. Menon, Active hyperbolic metamaterials: enhanced spontaneous emission and light extraction. Optica 2(1), 62-65 (2015)

160. L. Ferrari, J.S.T. Smalley, Y. Fainman, Z. Liu, Hyperbolic metamaterials for dispersion-assisted directional light emission. Nanoscale 9, 9034-9048 (2017)

161. D. Lu, L. Ferrari, J.J. Kan, E.E. Fullerton, Z. Liu, Optimization of nanopatterned multilayer hyperbolic metamaterials for spontaneous light emission enhancement. Phys. Status Solidi A 215(24), 1800263 (2018)

162. Afd. Mota, A. Martins, H. Ottevaere, W. Meulebroeck, E.R. Martins, J. Weiner, F.L. Teixeira, B.-H.V. Borges, Semianalytical model for design and analysis of grating-assisted radiation emission of quantum emitters in hyperbolic metamaterials. ACS Photonics 5(5), 1951-1959 (2018)

163. L. Ferrari, D. Lu, D. Lepage, Z. Liu, Enhanced spontaneous emission inside hyperbolic metamaterials. Opt. Express 22(4), 4301-4306 (2014)

164. D.J. Roth, A.V. Krasavin, A. Wade, W. Dickson, A. Murphy, S. Kéna-Cohen, R. Pollard, G.A. Wurtz, D. Richards, S.A. Maier, A.V. Zayats, Spontaneous 
emission inside a hyperbolic metamaterial waveguide. ACS Photonics $\mathbf{4}(10), 2513-2521$ (2017)

165. L. Li, W. Wang, T.S. Luk, X. Yang, J. Gao, Enhanced quantum dot spontaneous emission with multilayer metamaterial nanostructures. ACS Photonics 4(3), 501-508 (2017)

166. L. Wang, S. Li, B. Zhang, Y. Qin, Z. Tian, Y. Fang, Y. Li, Z. Liu, Y. Mei, Asymmetrically curved hyperbolic metamaterial structure with gradient thicknesses for enhanced directional spontaneous emission. ACS Appl. Mater. Interfaces 10(9), 7704-7708 (2018)

167. D. Lu, H. Qian, K. Wang, H. Shen, F. Wei, Y. Jiang, E.E. Fullerton, P.K.L. Yu, Z. Liu, Nanostructuring multilayer hyperbolic metamaterials for ultrafast and bright green InGaN quantum wells. Adv. Mater. 30(15), 1706411 (2018)

168. S. Li, P. Xu, Y. Xu, Local photonic density of states in hyperbolic metasurfaces. J. Opt. 23(11), 115101 (2021)

169. P. Tonkaev, S. Anoshkin, A. Pushkarev, R. Malureanu, M. Masharin, P. Belov, A. Lavrinenko, S. Makarov, Acceleration of radiative recombination in quasi-2D perovskite films on hyperbolic metamaterials. Appl. Phys. Lett. 118(9), 091104 (2021)

170. H.P. Adl, S. Gorji, M.K. Habil, I. Suarez, V.S. Chirvony, A.F. Gualdron-Reyes, I. Mora-Sero, L.M. Valencia, M. de la Mata, J. Hernandez-Saz et al., Purcell enhancement and wavelength shift of emitted light by $\mathrm{Cs} P$ bl3 perovskite nanocrystals coupled to hyperbolic metamaterials. ACS Photonics 7(11), 3152-3160 (2020)

171. G. Hu, C. Zheng, J. Ni, C.-W. Qiu, A. Alù, Enhanced light-matter interactions at photonic magic-angle topological transitions. Appl. Phys. Lett. 118(21), 211101 (2021)

172. S.-A. Biehs, M. Tschikin, P. Ben-Abdallah, Hyperbolic metamaterials as an analog of a blackbody in the near field. Phys. Rev. Lett. 109(10), 104301 (2012)

173. X. Liu, Z. Zhang, Giant enhancement of nanoscale thermal radiation based on hyperbolic graphene plasmons. Appl. Phys. Lett. 107(14), 143114 (2015)

174. I. Trushkov, I. Iorsh, Two-dimensional hyperbolic medium for electrons and photons based on the array of tunnel-coupled graphene nanoribbons. Phys. Rev. B 92(4), 045305 (2015)

175. C.J. Schuler, C. Wolff, K. Busch, M. Florescu, Thermal emission from finite photonic crystals. Appl. Phys. Lett. 95(24), 241103 (2009)

176. Y. Guo, C.L. Cortes, S. Molesky, Z. Jacob, Broadband super-Planckian thermal emission from hyperbolic metamaterials. Appl. Phys. Lett. 101(13), 131106 (2012)

177. Y. Guo, Z. Jacob, Thermal hyperbolic metamaterials. Opt. Express 21(12), 15014-15019 (2013)

178. P.N. Dyachenko, S. Molesky, A.Y. Petrov, M. Störmer, T. Krekeler, S. Lang, M. Ritter, Z. Jacob, M. Eich, Controlling thermal emission with refractory epsilon-near-zero metamaterials via topological transitions. Nat. Commun. 7, 11809 (2016)

179. Q. Li, H. He, Q. Chen, B. Song, Radiative thermal diode via hyperbolic metamaterials. arXiv preprint arXiv:2109.03496 (2021)

180. J.J. García-Esteban, J. Bravo-Abad, J.C. Cuevas, Deep learning for the modeling and inverse design of radiative heat transfer. arXiv preprint arXiv:2109.03114 (2021)

181. B. Liu, S. Shen, Broadband near-field radiative thermal emitter/absorber based on hyperbolic metamaterials: Direct numerical simulation by the Wiener chaos expansion method. Phys. Rev. B 87(11), 115403 (2013)

182. S. Campione, F. Marquier, J.-P. Hugonin, A.R. Ellis, J.F. Klem, M.B. Sinclair, T.S. Luk, Directional and monochromatic thermal emitter from epsilonnear-zero conditions in semiconductor hyperbolic metamaterials. Sci. Rep. 6, 34746 (2016)

183. G. Palermo, K.V. Sreekanth, N. Maccaferri, G.E. Lio, G. Nicoletta, F. De Angelis, M. Hinczewski, G. Strangi, Hyperbolic dispersion metasurfaces for molecular biosensing. Nanophotonics 10(1), 295-314 (2021)

184. L. Jiang, S. Zeng, Z. Xu, Q. Ouyang, D.-H. Zhang, P.H.J. Chong, P. Coquet, S. He, K.-T. Yong, Multifunctional hyperbolic nanogroove metasurface for submolecular detection. Small 13(30), 1700600 (2017)

185. A. Kabashin, P. Evans, S. Pastkovsky, W. Hendren, G. Wurtz, R. Atkinson, R. Pollard, V. Podolskiy, A. Zayats, Plasmonic nanorod metamaterials for biosensing. Nat. Mater. 8(11), 867-871 (2009)

186. K.V. Sreekanth, Y. Alapan, M. ElKabbash, E. Ilker, M. Hinczewski, U.A. Gurkan, A. De Luca, G. Strangi, Extreme sensitivity biosensing platform based on hyperbolic metamaterials. Nat. Mater. 15(6), 621-627 (2016)
187. G. Palermo, G.E. Lio, M. Esposito, L. Ricciardi, M. Manoccio, V. Tasco, A. Passaseo, A. De Luca, G. Strangi, Biomolecular sensing at the interface between chiral metasurfaces and hyperbolic metamaterials. ACS Appl. Mater. Interfaces 12(27), 30181-30188 (2020)

188. K.V. Sreekanth, Q. Ouyang, S. Sreejith, S. Zeng, W. Lishu, E. Ilker, W. Dong, M. ElKabbash, Y. Ting, C.T. Lim et al., Phase-change-material-based low-loss visible-frequency hyperbolic metamaterials for ultrasensitive label-free biosensing. Adv. Opt. Mater. 7(12), 1900081 (2019)

189. F. Ding, Y. Cui, X. Ge, Y. Jin, S. He, Ultra-broadband microwave metamaterial absorber. Appl. Phys. Lett. 100(10), 103506 (2012)

190. D. Ji, H. Song, X. Zeng, H. Hu, K. Liu, N. Zhang, Q. Gan, Broadband absorption engineering of hyperbolic metafilm patterns. Sci. Rep. $\mathbf{4}$ 4498 (2014)

191. X. Yin, C. Long, J. Li, H. Zhu, L. Chen, J. Guan, X. Li, Ultra-wideband microwave absorber by connecting multiple absorption bands of two different-sized hyperbolic metamaterial waveguide arrays. Sci. Rep. 5, 15367 (2015)

192. Y. Cui, K.H. Fung, J. Xu, H. Ma, Y. Jin, S. He, N.X. Fang, Ultrabroadband light absorption by a sawtooth anisotropic metamaterial slab. Nano Lett. 12(3), 1443-1447 (2012)

193. H. Hu, D. Ji, X. Zeng, K. Liu, Q. Gan, Rainbow trapping in hyperbolic metamaterial waveguide. Sci. Rep. 3, 1249 (2013)

194. S. He, F. Ding, L. Mo, F. Bao, Light absorber with an ultra-broad flat band based on multi-sized slow-wave hyperbolic metamaterial thin-films. Prog. Electromagn. Res. 147, 69-79 (2014)

195. J. Zhou, A.F. Kaplan, L. Chen, L.J. Guo, Experiment and theory of the broadband absorption by a tapered hyperbolic metamaterial array. ACS Photonics 1(7), 618-624 (2014)

196. Y.-L. Liao, Y. Zhao, S. Wu, S. Feng, Wide-angle broadband absorber based on uniform-sized hyperbolic metamaterial. Opt. Mater. Express 8(9), 2484-2493 (2018)

197. Q. Liu, W. Wu, S. Lin, H. Xu, Y. Lu, W. Song, Non-tapered metamaterial emitters for radiative cooling to low temperature limit. Opt. Commun. 450, 246-251 (2019)

198. A.P. Raman, M Abou Anoma, L. Zhu, E. Rephaeli, S. Fan, Passive radiative cooling below ambient air temperature under direct sunlight. Nature 515(7528), 540-544 (2014)

199. D. Lee, M. Go, S. Son, M. Kim, T. Badloe, H. Lee, J.K. Kim, J. Rho, Subambient daytime radiative cooling by silica-coated porous anodic aluminum oxide. Nano Energy 79, 105426 (2021)

200. M. Kim, D. Lee, S. Son, Y. Yang, H. Lee, J. Rho, Visibly transparent radiative cooler under direct sunlight. Adv. Opt. Mater. 9(13), 2002226 (2021)

201. M. Kim, D. Lee, Y. Yang, J. Rho, Switchable diurnal radiative cooling by doped $\mathrm{VO}_{2}$. Opto-Electronic Adv. 4(5), 200006 (2021)

202. Q. Zhang, G. Hu, W. Ma, P. Li, A. Krasnok, R. Hillenbrand, A. Alù, C.-W. Qiu, Interface nano-optics with van der Waals polaritons. Nature $\mathbf{5 9 7}(7875)$, 187-195 (2021)

203. X. Ling, H. Wang, S. Huang, F. Xia, M.S. Dresselhaus, The renaissance of black phosphorus. Proc. Natl. Acad. Sci. USA 112(15), 4523-4530 (2015)

204. F. Xia, H. Wang, Y. Jia, Rediscovering black phosphorus as an anisotropic layered material for optoelectronics and electronics. Nat. Commun. 5 , 4458 (2014)

205. T. Low, R. Roldán, H. Wang, F. Xia, P. Avouris, L.M. Moreno, F. Guinea, Plasmons and screening in monolayer and multilayer black phosphorus. Phys. Rev. Lett. 113(10), 106802 (2014)

206. A. Nemilentsau, T. Low, G. Hanson, Anisotropic 2D materials for tunable hyperbolic plasmonics. Phys. Rev. Lett. 116(6), 066804 (2016)

207. D. Correas-Serrano, J. Gomez-Diaz, A.A. Melcon, A. Alù, Black phosphorus plasmonics: anisotropic elliptical propagation and nonlocalityinduced canalization. J. Opt. 18(10), 104006 (2016)

208. A.J. Frenzel, C.C. Homes, Q.D. Gibson, Y. Shao, K.W. Post, A. Charnukha, R.J. Cava, D.N. Basov, Anisotropic electrodynamics of type-II Weyl semimetal candidate WTe $e_{2}$. Phys. Rev. B 95(24), 245140 (2017)

209. C. Wang, S. Huang, Q. Xing, Y. Xie, C. Song, F. Wang, H. Yan, Van der Waals thin films of $\mathrm{WTe}_{2}$ for natural hyperbolic plasmonic surfaces. Nat. Commun. 11, $1158(2020)$

210. S. Dai, Z. Fei, Q. Ma, A. Rodin, M. Wagner, A. McLeod, M. Liu, W. Gannett, W. Regan, K. Watanabe et al., Tunable phonon polaritons in atomically thin van der Waals crystals of boron nitride. Science $\mathbf{3 4 3 ( 6 1 7 5 ) ,}$ $1125-1129$ (2014) 
211. F. Wang, C. Wang, A. Chaves, C. Song, G. Zhang, S. Huang, Y. Lei, Q. Xing, L. Mu, Y. Xie et al., Prediction of hyperbolic exciton-polaritons in monolayer black phosphorus. Nat. Commun. 12, 5628 (2021)

212. D. Basov, M. Fogler, F.G. De Abajo, Polaritons in van der Waals materials. Science 354(6309), aag1992 (2016)

213. T. Low, A. Chaves, J.D. Caldwell, A. Kumar, N.X. Fang, P. Avouris, T.F. Heinz, F. Guinea, L. Martin-Moreno, F. Koppens, Polaritons in layered twodimensional materials. Nat. Mater. 16(2), 182-194 (2017)

214. G. Hu, J. Shen, C.-W. Qiu, A. Alù, S. Dai, Phonon polaritons and hyperbolic response in van der Waals materials. Adv. Opt. Mater. 8(5), $1901393(2020)$

215. G. Álvarez-Pérez, T.G. Folland, I. Errea, J. Taboada-Gutiérrez, J. Duan, J. Martín-Sánchez, A.I. Tresquerres-Mata, J.R. Matson, A. Bylinkin, M. He et al., Infrared permittivity of the biaxial van der Waals semiconductor $\alpha-\mathrm{MoO}_{3}$ from near-and far-field correlative studies. Adv. Mater. 32(29), $1908176(2020)$

216. Z. Dai, G. Hu, G. Si, Q. Ou, Q. Zhang, S. Balendhran, F. Rahman, B.Y. Zhang, J.Z. Ou, G. Li et al., Edge-oriented and steerable hyperbolic polaritons in anisotropic van der Waals nanocavities. Nat. Commun. 11, 6086 (2020)

217. G. Hu, Q. Ou, G. Si, Y. Wu, J. Wu, Z. Dai, A. Krasnok, Y. Mazor, Q. Zhang, Q. Bao et al., Topological polaritons and photonic magic angles in twisted $\alpha-\mathrm{MoO}_{3}$ bilayers. Nature 582(7811), 209-213 (2020)

218. P. Guo, W. Huang, C.C. Stoumpos, L. Mao, J. Gong, L. Zeng, B.T. Diroll, Y. Xia, X. Ma, D.J. Gosztola et al., Hyperbolic dispersion arising from anisotropic excitons in two-dimensional perovskites. Phys. Rev. Lett. 121(12), $127401(2018)$

219. X. Wang, A.M. Jones, K.L. Seyler, V. Tran, Y. Jia, H. Zhao, H. Wang, L. Yang, $X . X u, F$. Xia, Highly anisotropic and robust excitons in monolayer black phosphorus. Nat. Nanotechnol. 10(6), 517-521 (2015)

220. A. Sternbach, S. Chae, S. Latini, A. Rikhter, Y. Shao, B. Li, D. Rhodes, B. Kim, P.J. Schuck, X. Xu et al., Programmable hyperbolic polaritons in van der Waals semiconductors. Science 371(6529), 617-620 (2021)

221. M. Schubert, R. Korlacki, S. Knight, T. Hofmann, S. Schöche, V. Darakchieva, E. Janzén, B. Monemar, D. Gogova, Q.-T. Thieu et al., Anisotropy, phonon modes, and free charge carrier parameters in monoclinic $\beta$-gallium oxide single crystals. Phys. Rev. B 93(12), 125209 (2016)

222. N. Passler, X. Ni, G. Hu, J. Matson, M. Wolf, M. Schubert, A. Alu, J. Caldwell, T. Folland, A. Paarmann, Hyperbolic Shear Polaritons in Low-Symmetry Crystals (Res, Sq, 2021)

223. S. Dai, Q. Ma, T. Andersen, A. Mcleod, Z. Fei, M. Liu, M. Wagner, K. Watanabe, T. Taniguchi, M. Thiemens et al., Subdiffractional focusing and guiding of polaritonic rays in a natural hyperbolic material. Nat. Commun. $\mathbf{6}$, $6963(2015)$

224. A. Bylinkin, M. Schnell, M. Autore, F. Calavalle, P. Li, J. Taboada-Gutièrrez, S. Liu, J.H. Edgar, F. Casanova, L.E. Hueso et al., Real-space observation of vibrational strong coupling between propagating phonon polaritons and organic molecules. Nat. Photonics 15(3), 197-202 (2021)

225. M. Autore, P. Li, I. Dolado, F.J. Alfaro-Mozaz, R. Esteban, A. Atxabal, F. Casanova, L.E. Hueso, P. Alonso-González, J. Aizpurua et al., Boron nitride nanoresonators for phonon-enhanced molecular vibrational spectroscopy at the strong coupling limit. Light Sci. Appl. 7(4), 17172-17172 (2018)

226. M. He, S.I. Halimi, T.G. Folland, S.S. Sunku, S. Liu, J.H. Edgar, D.N. Basov, S.M. Weiss, J.D. Caldwell, Guided mid-IR and near-IR light within a hybrid hyperbolic-material/silicon waveguide heterostructure. Adv. Mater. 33(11), 2004305 (2021)

227. D. Li, H. Ma, Q. Zhan, J. Liao, W.-Y. Yin, H. Chen, H. Qian, High-speed efficient on-chip electro-optic modulator based on midinfrared hyperbolic metamaterials. Phys. Rev. Appl. 16, 034002 (2021)

228. D. Lee, M. Kim, S. So, I. Kim, G. Yoon, K. Kim, J. Rho, Demonstration of a hyperlens-integrated microscope and super-resolution imaging. J. Vis. Exp. 127, e55968 (2017)

229. G. Hu, M. Wang, Y. Mazor, C.-W. Qiu, A. Alù, Tailoring light with layered and moiré metasurfaces. Trends Chem. 3(5), 342-358 (2021)

230. Q. Zhang, Q. Ou, G. Hu, J. Liu, Z. Dai, M.S. Fuhrer, Q. Bao, C.-W. Qiu, Hybridized hyperbolic surface phonon polaritons at $\alpha-\mathrm{MoO}_{3}$ and polar dielectric interfaces. Nano Lett. 21, 3112-3119 (2021)

231. J. Martín-Sánchez, J. Duan, J. Taboada-Gutiérrez, G. Álvarez-Pérez, K.V. Voronin, I. Prieto, W. Ma, Q. Bao, V.S. Volkov, R. Hillenbrand et al., Focusing of in-plane hyperbolic polaritons in van der Waals crystals with tailored infrared nanoantennas. arXiv preprint arXiv:2103.10852 (2021)
232. G. Hu, C.-W. Qiu, A. Alù, Twistronics for photons: opinion. Opt. Mater. Express 11(5), 1377-1382 (2021)

233. Z. Dai, G. Hu, Q. Ou, L. Zhang, F. Xia, F.J. Garcia-Vidal, C.-W. Qiu, Q. Bao, Artificial metaphotonics born naturally in two dimensions. Chem. Rev. 120(13), 6197-6246 (2020)

234. Y.U. Lee, C. Posner, J. Zhao, J. Zhang, Z. Liu, Imaging of cell morphology changes via metamaterial-assisted photobleaching microscopy. Nano Lett. 21(4), 1716-1721 (2021)

235. Y.U. Lee, S. Li, S.E. Bopp, J. Zhao, Z. Nie, C. Posner, S. Yang, X. Zhang, J. Zhang, Z. Liu, Unprecedented fluorophore photostability enabled by low-loss organic hyperbolic materials. Adv. Mater. 33(9), 2006496 (2021)

236. Y.U. Lee, J. Zhao, G.C. Mo, S. Li, G. Li, Q. Ma, Q. Yang, R. Lal, J. Zhang, Z. Liu, Metamaterial-assisted photobleaching microscopy with nanometer scale axial resolution. Nano Lett. 20(8), 6038-6044 (2020)

237. Y.U. Lee, K. Yim, S.E. Bopp, J. Zhao, Z. Liu, Low-loss organic hyperbolic materials in the visible spectral range: a joint experimental and firstprinciples study. Adv. Mater. 32(28), 2002387 (2020)

238. Y.U. Lee, O.P. Gaudin, K. Lee, E. Choi, V. Placide, K. Takaishi, T. Muto, P. André, A. Muranaka, M. Uchiyama et al., Organic monolithic natural hyperbolic material. ACS Photonics 6(7), 1681-1689 (2019)

239. H. Jiang, W. Liu, K. Yu, K. Fang, Y. Sun, Y. Li, H. Chen, Experimental verification of loss-induced field enhancement and collimation in anisotropic $\mu$-near-zero metamaterials. Phys. Rev. B 91(4), 045302 (2015)

240. Z. Guo, H. Jiang, K. Zhu, Y. Sun, Y. Li, H. Chen, Focusing and super-resolution with partial cloaking based on linear-crossing metamaterials. Phys. Rev. Appl. 10(6), 064048 (2018)

241. Y. Yang, Z. Jia, T. Xu, J. Luo, Y. Lai, Z.H. Hang, Beam splitting and unidirectional cloaking using anisotropic zero-index photonic crystals. Appl. Phys. Lett. 114(16), 161905 (2019)

242. Y. Yang, P. Qin, X. Lin, E. Li, Z. Wang, B. Zhang, H. Chen, Type-I hyperbolic metasurfaces for highly-squeezed designer polaritons with negative group velocity. Nat. Commun. 10, 2002 (2019)

243. S. Hu, S. Du, J. Li, C. Gu, Multidimensional image and beam splitter based on hyperbolic metamaterials. Nano Lett. 21(4), 1792-1799 (2021)

244. H. Li, W. Hao, X. Yin, S. Chen, L. Chen, Broadband generation of airy beams with hyperbolic metamaterials. Adv. Opt. Mater. 7(20), 1900493 (2019)

245. L. Chen, H. Li, W. Hao, X. Yin, J. Wang, Hyperbolic metamaterials for highefficiency generation of circularly polarized Airy beams. Chin. Phys. B 29(8), 084210 (2020)

246. N. Maccaferri, Y. Zhao, T. Isoniemi, M. larossi, A. Parracino, G. Strangi, F. De Angelis, Hyperbolic meta-antennas enable full control of scattering and absorption of light. Nano Lett. 19(3), 1851-1859 (2019)

247. L. Shen, X. Lin, M.Y. Shalaginov, T. Low, X. Zhang, B. Zhang, H. Chen, Broadband enhancement of on-chip single-photon extraction via tilted hyperbolic metamaterials. Appl. Phys. Rev. 7(2), 021403 (2020)

248. P. Sohr, D. Wei, S. Tomasulo, M.K. Yakes, S. Law, Simultaneous large mode index and high quality factor in infrared hyperbolic metamaterials. ACS Photonics 5(10), 4003-4008 (2018)

249. K.-C. Shen, C.-T. Ku, C. Hsieh, H.-C. Kuo, Y.-J. Cheng, D.P. Tsai, Deep-ultraviolet hyperbolic metacavity laser. Adv. Mater. 30(21), 1706918 (2018)

250. S.V. Zhukovsky, A.A. Orlov, V.E. Babicheva, A.V. Lavrinenko, J. Sipe, Photonic-band-gap engineering for volume plasmon polaritons in multiscale multilayer hyperbolic metamaterials. Phys. Rev. A 90(1), 013801 (2014)

251. O. Shramkova, G. Tsironis, Propagation of electromagnetic waves in $P$ T-symmetric hyperbolic structures. Phys. Rev. B 94(3), 035141 (2016)

252. A. Calzolari, A. Catellani, M. Buongiorno Nardelli, M. Fornari, Hyperbolic metamaterials with extreme mechanical hardness. Adv. Opt. Mater. 9 2001904 (2021)

253. B.F. Diaz-Valencia, Extraordinary transverse magneto-optical Kerr effect through excitation of bulk plasmon polariton modes in type II magneto-optical hyperbolic metamaterials. Opt. Lett. 46(19), 4863-4866 (2021)

254. S. Wicharn, P. Buranasiri, Hybrid nanowires for phase-matching of thirdharmonic generation in hyperbolic metamaterial. Appl. Opt. 60(28), 8744-8755 (2021)

255. W.-L. Gao, F.-Z. Fang, Y.-M. Liu, S. Zhang, Chiral surface waves supported by biaxial hyperbolic metamaterials. Light Sci. Appl. 4(9), e328 (2015) 
256. S. Basak, O. Bar-On, J. Scheuer, Perovskite/metal-based hyperbolic metamaterials: tailoring the permittivity properties of coexisting anisotropies in the visible region. Adv. Opt. Mater. 9(1), 2001305 (2021)

257. Z. Jacob, E.E. Narimanov, Optical hyperspace for plasmons: Dyakonov states in metamaterials. Appl. Phys. Lett. 93(22), 221109 (2008)

258. A. Samusev, I. Mukhin, R. Malureanu, O. Takayama, D.V. Permyakov, I.S. Sinev, D. Baranov, O. Yermakov, I.V. Iorsh, A.A. Bogdanov, A.V. Lavrinenko, Polarization-resolved characterization of plasmon waves supported by an anisotropic metasurface. Opt. Express 25(26), 32631-32639 (2017)

259. O. Takayama, E. Shkondin, A. Bodganov, ME Aryaee. Panah, K. Golenitskii, P. Dmitriev, T. Repan, R. Malureanu, P. Belov, F. Jensen et al., Midinfrared surface waves on a high aspect ratio nanotrench platform. ACS Photonics 4(11), 2899-2907 (2017)

260. O. Takayama, P. Dmitriev, E. Shkondin, O. Yermakov, M. Panah, K. Golenitskii, F. Jensen, A. Bogdanov, A. Lavrinenko, Experimental observation of Dyakonov plasmons in the mid-infrared. Semiconductors 52(4), 442-446 (2018)

261. R. Li, C. Cheng, F.-F. Ren, J. Chen, Y.-X. Fan, J. Ding, H.-T. Wang, Hybridized surface plasmon polaritons at an interface between a metal and a uniaxial crystal. Appl. Phys. Lett. 92(14), 141115 (2008)

262. T. Repän, O. Takayama, A. Lavrinenko, Hyperbolic surface waves on anisotropic materials without hyperbolic dispersion. Opt. Express 28(22), 33176-33183 (2020)

263. Y. Liu, C. Ouyang, P. Zheng, J. Ma, Q. Xu, X. Su, Y. Li, Z. Tian, J. Gu, L. Liu et al., Simultaneous manipulation of electric and magnetic surface waves by topological hyperbolic metasurfaces. ACS Appl. Electron. Mater. 3(9), 4203-9 (2021)

264. W. Gao, M. Lawrence, B. Yang, F. Liu, F. Fang, B. Béri, J. Li, S. Zhang, Topological photonic phase in chiral hyperbolic metamaterials. Phys. Rev. Lett. 114, 037402 (2015)

265. B. Yang, Q. Guo, B. Tremain, L.E. Barr, W. Gao, H. Liu, B. Béri, Y. Xiang, D. Fan, A.P. Hibbins et al., Direct observation of topological surface-state arcs in photonic metamaterials. Nat. Commun. 8, 97 (2017)

266. M. Kim, W. Gao, D. Lee, T. Ha, T.-T. Kim, S. Zhang, J. Rho, Extremely broadband topological surface states in a photonic topological metamaterial. Adv. Opt. Mater. 7(20), 1900900 (2019)

267. L.-Z. Yin, F.-Y. Han, J. Zhao, D. Wang, T.-J. Huang, P.-K. Liu, Constructing Hyperbolic Metamaterials with Arbitrary Medium. ACS Photonics 8(4), 1085-1096 (2021)

268. I. Murataj, M. Channab, E. Cara, C.F. Pirri, L. Boarino, A. Angelini, F. Ferrarese Lupi, Hyperbolic metamaterials via hierarchical block copolymer nanostructures. Adv. Opt. Mater. 9(7), 2001933 (2021)

269. P. Gu, Y. Guo, J. Chen, Z. Zhang, Z. Yan, F. Liu, C. Tang, W. Du, Z. Chen, Multiple sharp fano resonances in a deep-subwavelength spherical hyperbolic metamaterial cavity. Nanomaterials 11(9), 2301 (2021)

270. M. Baqir, P. Choudhury, Q. Naqvi, Quasistatic analysis of hyperbolic metamaterial supported core-shell nanoparticle. Optik 246, 167850 (2021)

271. Z. Zheng, F. Sun, W. Huang, J. Jiang, R. Zhan, Y. Ke, H. Chen, S. Deng, Phonon polaritons in twisted double-layers of hyperbolic van der Waals crystals. Nano Lett. 20(7), 5301-5308 (2020)

272. J.A. Roberts, S.-J. Yu, P.-H. Ho, S. Schoeche, A.L. Falk, J.A. Fan, Tunable hyperbolic metamaterials based on self-assembled carbon nanotubes. Nano Lett. 19(5), 3131-3137 (2019)

273. S. Chen, E.S. Kang, M.S. Chaharsoughi, V. Stanishev, P. Kühne, H. Sun, C. Wang, M. Fahlman, S. Fabiano, V. Darakchieva et al., Conductive polymer nanoantennas for dynamic organic plasmonics. Nat. Nanotechnol. 15(1), 35-40 (2020)

274. D. Lee, M. Go, M. Kim, J. Jang, C. Choi, J.K. Kim, J. Rho, Multiple-patterning colloidal lithography-implemented scalable manufacturing of heat-tolerant titanium nitride broadband absorbers in the visible to near-infrared. Microsyst. Nanoeng. 7, 14 (2021)

275. W. Jung, Y.-H. Jung, P.V. Pikhitsa, J. Feng, Y. Yang, M. Kim, H.-Y.Tsai, T. Tanaka, J. Shin, K.-Y. Kim et al., Three-dimensional nanoprinting via charged aerosol jets. Nature 592, 54-59 (2021)

276. M. Lee, E. Lee, S. So, S. Byun, J. Son, B. Ge, H. Lee, H. Park, W. Shim, J. Pee, B. Min, S.-P. Cho, Z. Shi, T. Noh, J. Rho, J.-Y. Kim, I. Chung, Bulk metamaterials exhibiting chemically tunable hyperbolic responses. J. Am. Chem. Soc. (2021). https://doi.org/10.1021/jacs.1c08446 